\title{
The NADPH Oxidase A of Verticillium dahliae Is Essential for Pathogenicity, Normal Development, and Stress Tolerance, and It Interacts with Yap1 to Regulate Redox Homeostasis
}

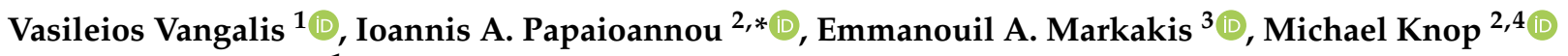 \\ and Milton A. Typas ${ }^{1, *}$ \\ 1 Department of Genetics \& Biotechnology, Faculty of Biology, National and Kapodistrian University of Athens, \\ 15784 Athens, Greece; vasvagg@biol.uoa.gr \\ 2 Center for Molecular Biology of Heidelberg University (ZMBH), University of Heidelberg, \\ 69120 Heidelberg, Germany; m.knop@zmbh.uni-heidelberg.de \\ 3 Laboratory of Mycology, Department of Viticulture, Vegetable Crops, Floriculture and Plant Protection, \\ Institute of Olive Tree, Subtropical Crops and Viticulture, N.AG.RE.F., Hellenic Agricultural \\ Organization-DIMITRA, 71307 Heraklion, Crete, Greece; markakis@elgo.iosv.gr \\ 4 German Cancer Research Center (DKFZ), DKFZ-ZMBH Alliance, 69120 Heidelberg, Germany \\ * Correspondence: i.papaioannou@zmbh.uni-heidelberg.de (I.A.P.); matypas@biol.uoa.gr (M.A.T.)
}

check for updates

Citation: Vangalis, V.; Papaioannou, I.A.; Markakis, E.A.; Knop, M.; Typas, M.A. The NADPH Oxidase A of Verticillium dahliae Is Essential for Pathogenicity, Normal Development, and Stress Tolerance, and It Interacts with Yap1 to Regulate Redox Homeostasis. J. Fungi 2021, 7, 740. https://doi.org/10.3390/jof7090740

Academic Editor: Katrina

Maria Ramonell

Received: 19 August 2021

Accepted: 7 September 2021

Published: 9 September 2021

Publisher's Note: MDPI stays neutral with regard to jurisdictional claims in published maps and institutional affiliations.

Copyright: (c) 2021 by the authors. Licensee MDPI, Basel, Switzerland. This article is an open access article distributed under the terms and conditions of the Creative Commons Attribution (CC BY) license (https:// creativecommons.org/licenses/by/ $4.0 /)$.
Abstract: Maintenance of redox homeostasis is vital for aerobic organisms and particularly relevant to plant pathogens. A balance is required between their endogenous ROS production, which is important for their development and pathogenicity, and host-derived oxidative stress. Endogenous ROS in fungi are generated by membrane-bound NADPH oxidase (NOX) complexes and the mitochondrial respiratory chain, while transcription factor Yap1 is a major regulator of the antioxidant response. Here, we investigated the roles of NoxA and Yap1 in fundamental biological processes of the important plant pathogen Verticillium dahliae. Deletion of noxA impaired growth and morphogenesis, compromised formation of hyphopodia, diminished penetration ability and pathogenicity, increased sensitivity against antifungal agents, and dysregulated expression of antioxidant genes. On the other hand, deletion of yap1 resulted in defects in conidial and microsclerotia formation, increased sensitivity against oxidative stress, and down-regulated antioxidant genes. Localized accumulation of ROS was observed before conidial fusion and during the heterokaryon incompatibility reaction upon nonself fusion. The frequency of inviable fusions was not affected by the deletion of Yap1. Analysis of a double knockout mutant revealed an epistatic relationship between nox $A$ and yap1. Our results collectively reveal instrumental roles of NoxA and ROS homeostasis in the biology of V. dahliae.

Keywords: antioxidant response; conidial anastomosis tubes (CATs); heterokaryon incompatibility; oxidative stress; pathogenicity; reactive oxygen species (ROS); redox homeostasis

\section{Introduction}

Reactive oxygen species (ROS) are short-lived, highly reactive molecules that are produced by partial reduction of oxygen, and they include hydrogen peroxide $\left(\mathrm{H}_{2} \mathrm{O}_{2}\right)$, superoxide anion $\left(\mathrm{O}_{2}{ }^{\bullet-}\right)$ and hydroxyl $\left(\mathrm{OH}^{\bullet}\right)$ radicals [1]. Growing evidence from various organisms has assigned a Janus-faced nature to ROS as both deleterious molecules that can cause irreversible damage to biological systems and important signaling components regulating metabolic and developmental processes [1-3]. This double-edged significance of ROS is clearly illustrated in host-microbe interactions. In particular, fungal plant pathogens have to cope with host-derived ROS burst reactions during infection [4], while endogenous ROS production is simultaneously involved in the development of infectious structures and the efficient colonization of their hosts [5]. It is, therefore, vital for fungal pathogens to 
maintain a balance between ROS generation and scavenging in order to achieve successful development and pathogenicity [5,6].

Intracellular ROS production mainly takes place in mitochondria, as by-products of the electron transport chain [7], and by the enzymatic activity of NADPH oxidase (Nox) complexes [1]. The latter mostly localize at the plasma membrane or at the endoplasmic reticulum, and they produce superoxide anion radicals via reduction of molecular oxygen, using NADPH as the electron donor [1]. The best characterized member of this family is the mammalian gp91 ${ }^{\text {phox }}$ (Nox2), which is responsible for the ROS burst of neutrophils and other phagocytic cells in response to microbial pathogens [8]. Filamentous fungi possess three well established families of Nox enzymes called NoxA, NoxB (homologs of the mammalian gp91 ${ }^{\text {phox }}$ ), and NoxC. These enzymes, together with their adapter protein NoxD, form distinct complexes with pleiotropic roles in sexual reproduction, vegetative growth, and host infection [5,6,9-13]. In particular, NoxA has been implicated in sexual differentiation of fruiting bodies in Aspergillus nidulans [14], Podospora anserina [15], and Sordaria macrospora [16], while its deletion in Neurospora crassa has led to complete female fertility [17]. Generation of ROS by NoxA is indispensable for appressorium-mediated cuticle penetration in Magnaporthe oryzae [10], as it controls the polarized elongation of penetrations pegs [11]. Similarly, a complete lack or high attenuation of pathogenicity was observed upon deletion of noxA in Claviceps purpurea [18], Sclerotinia sclerotiorum [19], Botrytis cinerea [20], and Alternaria alternata [21]. Finally, NoxA has been proposed to be involved in the control of somatic conidial fusion via conidial anastomosis tubes (CATs) in various species [22], including the plant pathogens Verticillium dahliae [23], Fusarium oxysporum [24], and B. cinerea [25].

In parallel with ROS-generating systems, fungi have evolved a fine-tuned arsenal to cope with oxidative stress. Basic leucine zipper (bzip) transcription factors are conserved across eukaryotic life and are implicated, among others, in the oxidative stress response (OSR), developmental processes, amino acid biosynthesis, and nutrient utilization [26,27]. The AP1 family is the largest and best characterized group of fungal bzip transcription factors [28], and it includes Yap1, the major regulator of the OSR, which orchestrates several ROS-scavenging enzymes and non-enzymatic antioxidants [5]. Extensive studies of Saccharomyces cerevisiae Yap1 revealed that oxidation of specific cysteine residues upon exposure to $\mathrm{H}_{2} \mathrm{O}_{2}$ is crucial for the accumulation of the protein in the nucleus, providing a characteristic example of ROS-mediated signal transduction [29]. Homologs of Yap1 in filamentous fungi have similar structures and their deletion increases sensitivity to oxidative agents, indicating the conserved role of Yap1 in the regulation of the OSR [28]. Transcriptional analyses in several species have shown that Yap1 homologs control the expression of antioxidant genes such as catalases, superoxide dismutases, peroxidases, and genes involved in glutathione biosynthesis [30-37]. Apart from their universal role in regulating the OSR, Yap1 homologs also exhibit species-specific roles in pathogenicity $[33,36,38,39]$, development $[33,37,39,40]$, and secondary metabolism $[35,41]$.

The asexual plant-pathogenic fungus $V$. dahliae causes wilt disease in a wide range of economically important plants [42]. Upon induction from a plant host, its resting structures (i.e., microsclerotia) in the soil germinate and form infectious hyphae that attach to a host's surface and penetrate its roots by forming swollen hyphae (termed hyphopodia) and penetration pegs [42,43]. The formation of these pegs depends on a ROS burst derived from the NoxB/Pls1 membrane-bound complex [44]. During host colonization, $V$. dahliae must cope with the plant-derived oxidative stress that is induced by plant defense mechanisms [45], which renders efficient OSRs necessary for successful systemic infection. However, a recent study of $V$. dahliae Yap1 showed that its deletion did not impair the ability of this fungus to cause disease in smoke trees [40].

In this work, we aimed at the functional characterization of nox $A$ and its potential involvement in important biological processes of $V$. dahliae. We coupled this investigation with further analyses of yap1, which has recently been studied in V. dahliae [40], to gain insight into the possible interplay between ROS-generating and -scavenging systems in 
fungi. To this end, we knocked out their $V$. dahliae homologs and further generated a double knockout mutant to investigate their roles in fungal development, physiology, and pathogenicity. Furthermore, we characterized the responses of these mutants to antifungal agents and their behavior during hyphal fusion and the heterokaryon incompatibility reaction. Our results attribute significant roles to NoxA and Yap1 regarding morphogenesis, pathogenicity, and stress tolerance, and they highlight the crucial contribution of endogenous ROS production and metabolism to multiple aspects of fungal biology.

\section{Materials and Methods}

\subsection{Fungal Strains, Growth Media, and Culture Conditions}

All fungal strains constructed and used in this study are listed in Table S1 (Supplementary Materials). They were grown on standard growth media (Potato Dextrose Agar-PDA, Czapek-Dox complete medium-CM, Czapek-Dox minimal medium-MM) at $24{ }^{\circ} \mathrm{C}$, in the dark. Preparation and maintenance of monoconidial strains have been described previously [46].

\subsection{Deletion and Complementation of $V$. dahliae nox $A$ and yap1}

All plasmids used for the construction of recombinant vectors are listed in Table S2 (Supplementary Materials). We have recently described the strategy that we used for knocking out the $V$. dahliae homologs of nox $A$ and yap1 [23]. Briefly, both mutants were constructed in the background of $V$. dahliae wild-type strain $123 \mathrm{~V}$ via double homologous recombination following Agrobacterium tumefaciens-mediated transformation (ATMT) [47]. Using this method, we recently performed and described deletion of noxA and validation of the mutant strain [23]. To knock out the yap1 homolog, the flanking chromosomal regions ( $2.0 \mathrm{~kb}$-long each) of the yap1 open reading frame (ORF) were amplified from genomic DNA of $V$. dahliae $123 \mathrm{~V}$ and ligated to the $n e o^{R}$ cassette (conferring resistance to geneticin; the cassette was amplified from plasmid pSD1 [48]) in the backbone of the A. tumefaciens binary vector pOSCAR [49]. All PCR amplification steps were performed using the high-fidelity Herculase II Fusion DNA Polymerase (Agilent, Santa Clara, CA, USA), and assembly of the recombinant vector was performed with the NEBuilder HiFi DNA Assembly Master Mix (New England Biolabs, Ipswich, MA, USA). To generate the double $\Delta$ noxA $\triangle$ yap1 knockout mutant, we transformed the $\triangle$ nox $A$ strain with the deletion construct of yap1. Validation of all knockout strains was achieved with PCR, using genespecific primers (Table S3, Supplementary Materials), and Southern hybridization analyses (DIG DNA Labeling and Detection Kit, Sigma-Aldrich, St. Louis, MO, USA).

The coding sequence of the yap1 gene, flanked by $\sim 2.0 \mathrm{~kb}$-long genomic regions, was amplified from genomic DNA of $V$. dahliae $123 \mathrm{~V}$ and co-transformed into protoplasts of the $\triangle$ yap1 strain with plasmid pUCATPH [50] (which carries the hph cassette conferring resistance to hygromycin B) to generate the complemented strain yap1-c.

\subsection{Characterization of Morphology, Physiology, and Stress Response of V. dahliae Strains}

Morphological and physiological characterization of fungal strains, as well as assessment of their stress sensitivity, were performed as previously described [47], with a minor modification for the determination of germination frequency. In particular, strains were grown for $12 \mathrm{~h}$ in $\mathrm{CM}$ and then checked microscopically for the emergence of germ tubes.

To characterize stress tolerance and responses, we exposed fungal strains to a variety of oxidative agents $\left(\mathrm{H}_{2} \mathrm{O}_{2}\right.$, paraquat, iprodione, and $\mathrm{N}$-acetyl cysteine), substances that induce osmotic stress ( $\mathrm{NaCl}$ and sorbitol), cell wall damaging factors (amphotericin $\mathrm{B}$ obtained from Biosera, Nuaille, France, fluconazole from Pfizer, Brooklyn, NY, USA, calcofluor white M2R, and Congo red), trace elements $\left(\mathrm{CaCl}_{2}, \mathrm{CuSO}_{4}\right.$, and $\left.\mathrm{FeSO}_{4}\right)$, and an inhibitor of TOR kinase (sirolimus from Cayman Chemical, Ann Arbor, MI, USA). In addition, we used fungicides that target complex I (sodium amytal), complex II (isopyrazam, flutolanil), complex III (azoxystrobin, kresoxim-methyl, pyraclostrobin-QoI/cytB site; annisulbornQi site), and complex IV (sodium cyanide) of the electron transport chain to investigate 
possible interactions between the ROS-generating systems. All chemicals were purchased from Sigma-Aldrich, St. Louis, MO, USA, unless otherwise specified above. According to our previously described methods [47], we used spot dilution assays and determined relative growth inhibition (i.e., $\%$ growth inhibition $=(($ colony diameter on $\mathrm{CM}-$ colony diameter in stress condition) $/($ colony diameter on $C M)) \times 100)$. All experiments were performed at least in triplicate.

\subsection{Virulence Assays}

Plant pathogenicity bioassays were performed according to our previously published methods $[47,51]$. Briefly, eggplant seedlings were inoculated with the corresponding fungal strain by soil drenching ( $20 \mathrm{~mL}$ of conidial suspension of $5.0 \times 10^{6}$ conidia $/ \mathrm{mL}$ per pot). Plants were maintained at $24{ }^{\circ} \mathrm{C}$ with a $12 \mathrm{~h}$ light/dark cycle. Assessment of disease severity at each time point (up to 40 days) and determination of relative AUDPC and plant growth parameters were performed according to previously described protocols [51,52]. We re-isolated fungal strains from the treated plants by transferring xylem chips (three chips from nine randomly selected plants per treatment) to acidified PDA plates. The isolation ratio was expressed as the number of xylem chips from each treatment that exhibited fungal growth.

\subsection{Cellophane Penetration Assays}

The ability of $V$. dahliae strains to penetrate cellophane membranes was assessed as follows. Conidial suspensions from the examined strains were collected from 7-day-old PDA cultures, and $5 \times 10^{6}$ conidia of each were transferred onto CM plates overlaid with sterile cellophane sheets. The samples were incubated at $24^{\circ} \mathrm{C}$ for five days. Cellophane sheets were then removed, and the plates were incubated for four additional days before being scored for fungal growth. For the microscopic examination of hyphopodial formation, the same membranes (i.e., after five days of incubation with the respective conidial suspension) were washed with sterile water and observed under a microscope.

\subsection{Superoxide Detection}

Superoxide anion radicals $\left(\mathrm{O}_{2}{ }^{--}\right)$in germlings and in the mycelium of $V$. dahliae strains were detected by a nitro blue tetrazolium chloride (NBT; Cayman Chemical, Ann Arbor, MI, USA) staining assay. Conidia of each strain were grown for $16 \mathrm{~h}$ in CM, in plate wells with sterile coverslips at their bottom. Following incubation, the medium was removed, $5 \mathrm{~mL}$ of a $0.2 \%$ NBT solution was added, and the samples were incubated at room temperature (RT), in the dark, for $45 \mathrm{~min}$. The coverslips were then washed with ethanol and checked microscopically. Detection of superoxide anion radicals in the mycelium was performed with the addition of $10 \mathrm{~mL}$ of a $0.2 \%$ NBT solution on 20-day-old cultures grown on CM. Plates were incubated for $45 \mathrm{~min}$ at RT, which was followed by washing with ethanol and incubation for another $30 \mathrm{~min}$, before being air-dried and scored. Staining assays were performed in duplicate.

\subsection{Reverse Transcription Quantitative PCR (RT- $q P C R)$}

Determination of the expression levels of selected genes was performed as follows. Wild-type, $\triangle$ noxA, $\triangle y a p 1$, and $\triangle$ noxA $\triangle$ yap 1 strains were grown for five days in $\mathrm{CM}$ at $24{ }^{\circ} \mathrm{C}$. Mycelia were collected, washed with water, and when desired treated with $1.5 \mathrm{mM} \mathrm{H}_{2} \mathrm{O}_{2}$ for $45 \mathrm{~min}$. They were then snap-frozen in liquid nitrogen $\left(\mathrm{N}_{2}\right)$, ground to a fine powder under $\mathrm{N}_{2}$, and used for extraction of total RNA with the NucleoSpin RNA kit (Macherey-Nagel, Düren, Germany). Total RNA was reverse transcribed to cDNA using the PrimeScript cDNA synthesis kit (Takara Bio, Kusatsu, Japan). Quantitative PCR (qPCR) was performed using the KAPA SYBR Fast Universal Master Mix (Roche, Basel, Switzerland) in a Mx3000P real-time PCR instrument (Stratagene California, San Diego, CA, USA), according to the manufacturer's instructions. For each primer pair (Table S3, Supplementary Materials), we performed optimization of oligo concentrations 
and construction of standard curves in preliminary experiments. All curves were highly linear $\left(R^{2}>0.999\right)$, and their amplification efficiencies ranged between $95 \%$ and $105 \%$. The cycling protocol consisted of an initial denaturation step at $95^{\circ} \mathrm{C}$ for $5 \mathrm{~min}$, followed by 40 cycles of $10 \mathrm{~s}$ at $95^{\circ} \mathrm{C}$ (denaturation), $20 \mathrm{~s}$ at $60^{\circ} \mathrm{C}$ (annealing), and $20 \mathrm{~s}$ at $72{ }^{\circ} \mathrm{C}$ (elongation). Dissociation curves were generated for each primer pair with the following protocol. Samples were first incubated for $1 \mathrm{~min}$ at $95^{\circ} \mathrm{C}$, then at $55^{\circ} \mathrm{C}$ for $30 \mathrm{~s}$, and finally a temperature ramp $\left(0.1^{\circ} \mathrm{C} / \mathrm{s}\right)$ up to $95^{\circ} \mathrm{C}$ was applied with continuous collection of fluorescence readings. A single product was amplified with each primer pair. No-template control samples were included in each run to check for contamination and significant formation of primer dimers.

The $2^{-\Delta \Delta C t}$ method [53] was used for analysis of the results, with the modification that each $\Delta \mathrm{Ct}$ value was calculated as the difference between the $\mathrm{Ct}$ values of the reference and target genes (i.e., $\mathrm{Ct}$ reference-Ct target), and the fold change was calculated by the formula $2^{\Delta \Delta \mathrm{Ct}}$. The $V$. dahliae $\beta$-tubulin gene (VDAG_10074) was used as the internal reference in all experiments. The $\Delta \Delta \mathrm{Ct}$ value and fold change for each gene in different mutants and/or conditions was calculated relative to the control condition (i.e., expression level of the corresponding gene in the untreated wild-type strain). Student's $t$-tests were used to assess differences of $\Delta \Delta \mathrm{Ct}$ values. Three biological (i.e., independent cultures) and two technical replicates were performed for each strain and condition.

\subsection{Quantification of CAT-Mediated Fusion}

We used our previously optimized and described methods for reproducible quantification of CAT-mediated self and nonself fusion $[23,54]$ to investigate the possible involvement of noxA and yap 1 in somatic cell fusion. Briefly, conidia from 7-day-old PDA cultures were collected and transferred to 6-mm Petri dishes, with coverslips at their bottom (containing $5 \mathrm{~mL}$ of CAT medium each) to a final concentration of $2.0 \times 10^{6}$ conidia $/ \mathrm{mL}$. Plates were incubated for $60 \mathrm{~h}$ at $24^{\circ} \mathrm{C}$ before imaging. Each strain/pairing was tested in triplicate and 200 fusions were recorded per replicate.

\subsection{Microscopy}

Microscopic examination of fungal germlings, hyphae, and NBT-stained samples, as well as investigation of CAT-mediated fusion, were performed using a Zeiss Axioplan epifluorescence microscope equipped with a differential interference contrast (DIC) optical system, a set of filters BP450-490 (excitation) and BP515-595 (emission), and a Zeiss Axiocam MRc5 digital camera. Methylene blue staining $(0.005 \% w / v)$ was used to differentiate live from dead cells; samples were incubated at $25^{\circ} \mathrm{C}$ for $5 \mathrm{~min}$ (in the dark) before imaging.

\section{Results}

\subsection{Roles of NoxA and Yap1 in V. dahliae Morphogenesis and Physiology}

The NADPH oxidase A (NoxA) and the transcriptional regulator Yap1 are key components of ROS metabolism in fungi and other organisms. The single homolog of noxA in the $V$. dahliae genome (VDAG_06812 in the reference genome of strain Ls.17) codes for a predicted protein of 555 aa with a high similarity to its N. crassa Nox1 homolog (99\% query coverage, $90 \%$ sequence similarity), and we have recently described its deletion in the wild-type $V$. dahliae strain $123 \mathrm{~V}$ [23]. The $A$. nidulans Yap1 protein sequence was used as a query in tBlast $N$ genomic searches for the identification of its single $V$. dahliae homolog, which encodes a predicted protein of 583 aa (VDAG_01588; protein similarity: 52\%). This protein is predicted to contain the characteristic bzip domain and the signal peptides of its S. cerevisiae homolog, as well as the conserved cysteine residues that are essential for its nuclear localization (Figure S1, Supplementary Materials). For the functional analysis of yap 1 in $V$. dahliae, the gene was deleted from strain $123 \mathrm{~V}$, and the resulting deletion mutant ( $\triangle$ yap1) was validated by PCR and Southern blot analyses (Figure S2, Supplementary Materials). The wild-type yap 1 gene was re-introduced into $\Delta$ yap 1 to generate the complemented strain yap1-c. A double deletion $\Delta$ noxA $\triangle$ yap1 mutant was constructed via 
double homologous recombination following transformation of $\Delta n o x A$ conidia with the yap1 deletion construct (Figure S2, Supplementary Materials).

Morphogenesis and physiology of strains $\Delta$ noxA, $\Delta$ yap1, and $\Delta$ noxA $\triangle$ yap1 were compared to those of their wild type on growth media PDA, CM, and MM. Production of microsclerotia was significantly reduced in mutants $\triangle$ noxA and $\triangle$ noxA $\triangle$ yap 1 on PDA, while it was almost absent from $\triangle y a p 1$ on all media (Figure 1A). The ability of $\triangle n o x A$ and $\triangle$ noxA $\triangle$ yap 1 to produce aerial hyphae was compromised on CM and MM (Figure 1A), and these strains also exhibited moderately slower growth on PDA $(p<0.01$ and $p<0.05$, respectively; Figure 1B). In contrast, deletion of yap1 did not affect the formation of aerial mycelium, but it slightly reduced its growth rate $(p<0.05$, Figure $1 \mathrm{~A}, \mathrm{~B})$.

Regarding development of conidia, a non-significant increase in their production was observed in $\triangle$ noxA, but their ability to germinate was drastically limited in this mutant (51.3\% reduction), similarly to what was observed in $\Delta$ nox $A \Delta y a p 1$ (43.7\% reduction) (Figure 1B). On the other hand, $\triangle$ yap1 exhibited a 10-fold reduction in conidiation (Figure 1B), although its conidia germinated normally (Figure 1B,C). We also observed that mature hyphae of $\Delta$ nox $A$ and $\triangle$ noxA $\triangle$ yap 1 were significantly thinner than those of the wild type (Figure 1D,E). All these defects were fully rescued in the corresponding complemented strains (Figure 1A,B,E).

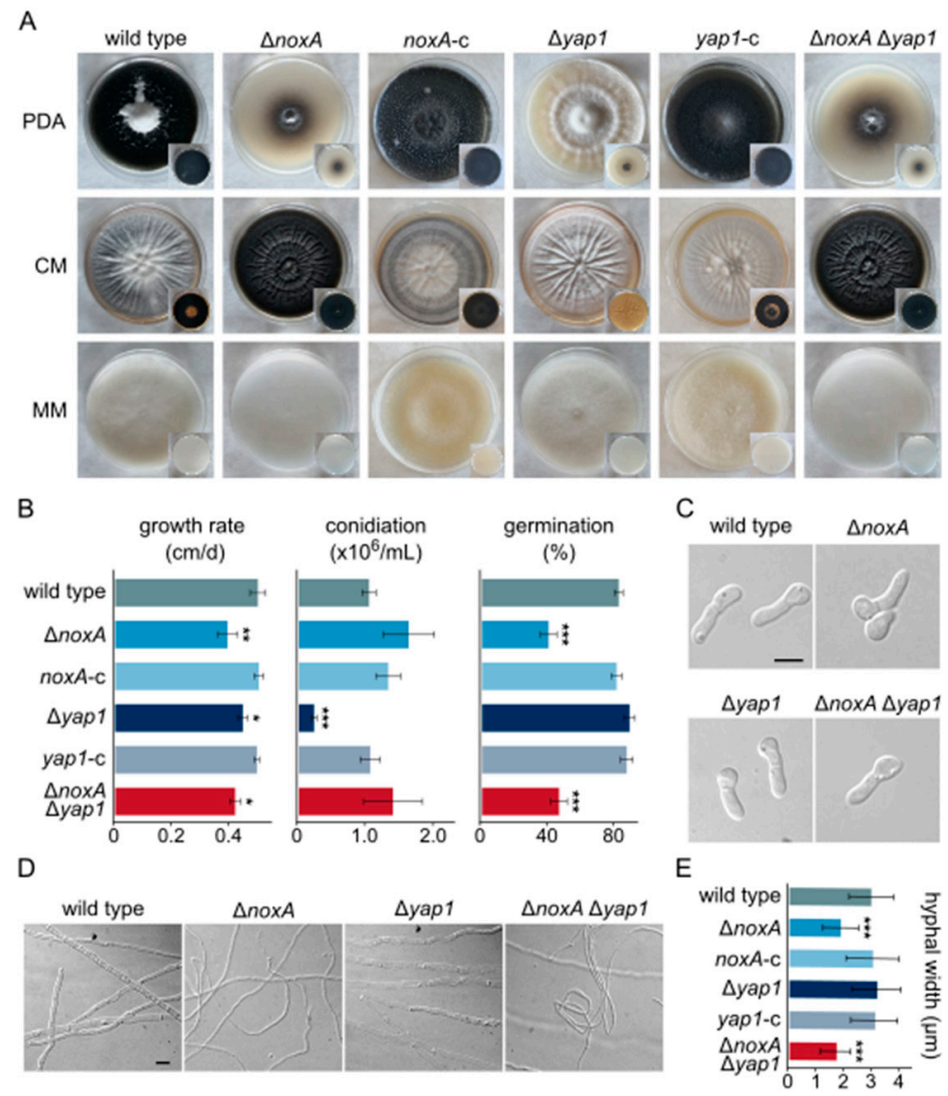

Figure 1. Morphological and physiological characterization of $V$. dahliae $\Delta$ noxA, $\Delta y a p 1$, and $\Delta$ noxA $\Delta$ yap1 deletion mutants: (A) morphology of colonies of the $V$. dahliae wild-type (123V), $\Delta$ noxA, $\Delta y a p 1$, $\triangle$ noxA $\triangle$ yap1, and the complemented strains noxA-c and yap1-c after growth on PDA, CM, or MM for 35 days. (B) Growth rate, conidial production, and germination frequency of tested strains. All experiments were performed in triplicate, and germination of 100 conidia was assessed per replicate. Bars: SD. Statistical significance of differences from the wild type were tested with Student's $t$-tests $\left({ }^{*} p \leq 0.05,{ }^{* *} p \leq 0.01,{ }^{* *} p \leq 0.001\right)$. (C) Morphology of conidial germlings of the wild-type and the knockout strains. Bar $=5 \mu \mathrm{m}$. (D) Morphology of mature hyphae. Bar $=5 \mu \mathrm{m}$. (E) Mean hyphal width of examined strains. For each strain, the widths of at least 50 non-apical hyphal compartments from colony areas of the same age were recorded. Bars: SD. Statistical significance of differences from the wild type was assessed using Student's $t$-tests $(* * * 00.001)$. 


\subsection{NoxA, but Not Yap1, Is Essential for the Penetration Ability and Pathogenicity of V. dahliae}

To investigate the possible involvement of NoxA and Yap1 in V. dahliae pathogenicity, we assessed the ability of the deletion mutants to cause disease in eggplant, in comparison to their wild type. Both $\triangle$ noxA and $\triangle$ noxA $\triangle$ yap 1 mutants exhibited diminished pathogenicity, with $88 \%$ and $76 \%$ of the treated plants (respectively) remaining completely asymptomatic 40 days after inoculation, while the remaining plants showed only weak symptoms (Figure 2A,B). In contrast, deletion of yap1 only slightly limited the fungal potential to cause disease (Figure 2A,B). Complementation of the corresponding mutants with the wild-type alleles of noxA and yap 1 fully rescued the ability of the fungus to cause severe disease, characterized by defoliation, wilting, and chlorosis (Figure 2A,B).

A

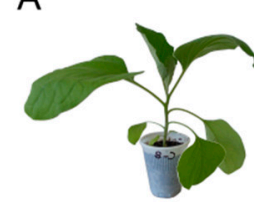

mock

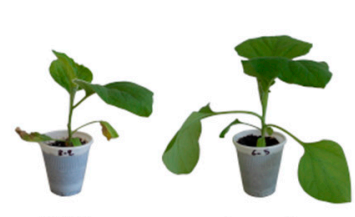

WT

$\triangle \operatorname{nox} A$

B

$\%$ disease severity $=0$ =1-20 $=21-50=51-79=80-100$

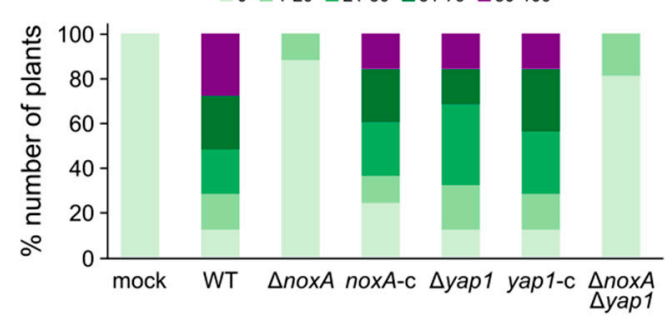

D

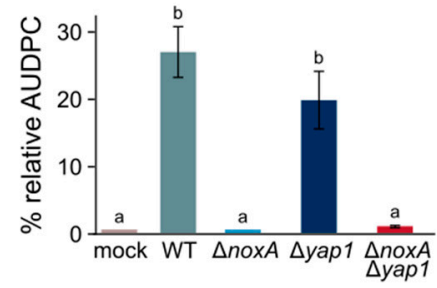

$\mathrm{E}$

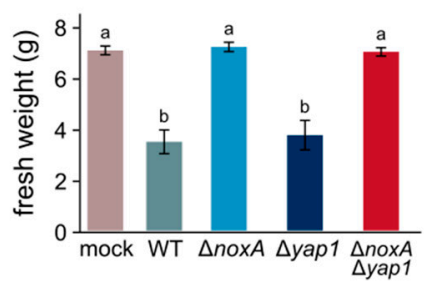

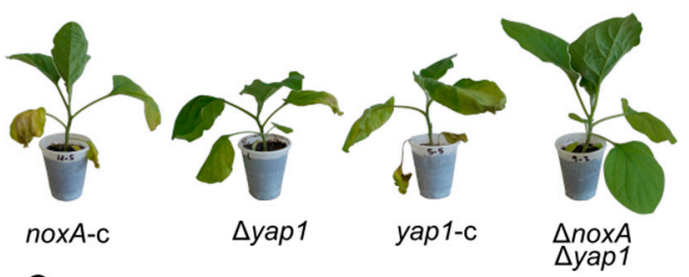

$\mathrm{C}$

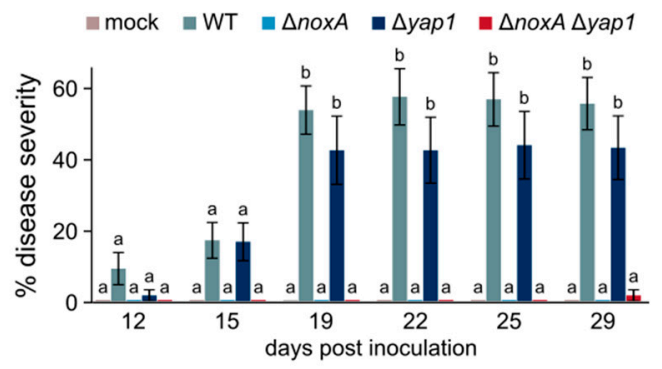

$\mathrm{F}$

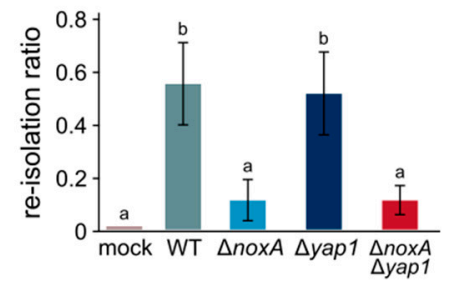

Figure 2. Phytopathological characterization of $V$. dahliae noxA and yap1 knockout strains: (A) representative examples of inoculated plants with the wild-type strain $123 \mathrm{~V}(\mathrm{WT})$, the deletion mutants $\triangle$ noxA, $\triangle y a p 1$, and $\triangle$ noxA $\triangle$ yap 1 , as well as their corresponding complemented strains, 29 days post-inoculation. (B) Average disease severity caused by the examined strains, 40 days after inoculation (30 eggplant seedlings/strain). Non-infected plants (mock) served as controls. (C) Time-course analysis of disease severity caused by the wild-type and the knockout strains (21 plants/strain) over 29 days. (D) Mean relative area under disease progress curve (AUDPC) score of each strain (29 days). (E) Average plant fresh weight at the end of the time-course experiment (29 days). (F) Fungal re-isolation ratios at the end of the time-course experiment (29 days). Bars in (C-F): SE. Statistical significance of differences between strains was tested by one-way ANOVA followed by Tukey's post hoc tests. Bars marked with the same letter did not differ significantly $(p \leq 0.05)$.

We further performed a time-course analysis of virulence to gain a better understanding of the involvement of noxA and yap 1 in the induction of plant disease (Figure 2C). The wild-type strain started causing observable symptoms in the infected plants 12 days after inoculation, reaching a mean disease severity of $55.5 \%( \pm 7.3 \%)$ at the end of the experiment (29 days), and an overall relative AUDPC value of $27.5 \%$ ( $\pm 3.9 \%$ ) (Figure $2 \mathrm{C}, \mathrm{D})$. Deletion of noxA completely prevented the expression of disease symptoms during this time pe- 
riod, similarly to the $\triangle$ noxA $\triangle y a p 1$ strain, which achieved a relative AUDPC score of $0.1 \%$ $( \pm 0.1 \%)$ (Figure $2 \mathrm{C}, \mathrm{D})$. In contrast, only a minor reduction was detected in virulence of $\Delta y a p 1$ (Figure 2C,D). These findings were further supported by the determination of the average plant fresh weight at the end of the experiment, with $\triangle$ nox $A$ and $\triangle$ nox $A \triangle y a p 1$ having no effect, whereas $\triangle$ yap1 led to a reduction similar to that of the wild-type strain (Figure 2E). Consistently, our attempts to re-isolate the fungus from xylem chips (29 days post-inoculation) revealed a significantly reduced presence of the pathogen in the xylem vessels of plants inoculated with $\triangle$ noxA or $\triangle$ noxA $\triangle y a p 1$, in contrast to $\triangle y a p 1$, which achieved systemic colonization at wild-type levels (Figure $2 \mathrm{~F}$ ).

The inability of the $\triangle$ nox $A$ and $\triangle$ nox $A \Delta y a p 1$ strains to cause disease in their plant hosts, as well as their severely reduced presence in the xylem of inoculated plants, led us to the hypothesis that nox $A$ is possibly necessary for efficient root penetration. We therefore investigated the capacity of the deletion mutants to penetrate cellophane membranes by inoculating cellophane sheets overlaying CM plates with conidia of each strain and incubating them for 5 days. Cellophane sheets were then removed, plates were incubated for another 4 days, and they were finally scored for fungal growth. In support of our hypothesis, deletion of nox $A$, or both nox $A$ and yap1, completely abolished penetration of cellophane, in contrast to the wild-type, the $\Delta y a p 1$, and the complemented strains, which grew profoundly on but also below the membrane sheets (Figure 3A). Furthermore, microscopic examination of the removed membranes revealed a drastic reduction in the frequency of normally developed hyphopodia (i.e., infectious structures involved in host penetration [43]) in $\triangle$ nox $A$ and $\triangle$ noxA $\triangle y a p 1$, whereas all other strains retained the ability to form numerous hyphopodia (Figure 3B). Our results indicate that NoxA, but not Yap1, plays a major role in plant penetration and has essential functions in $V$. dahliae pathogenicity.

A

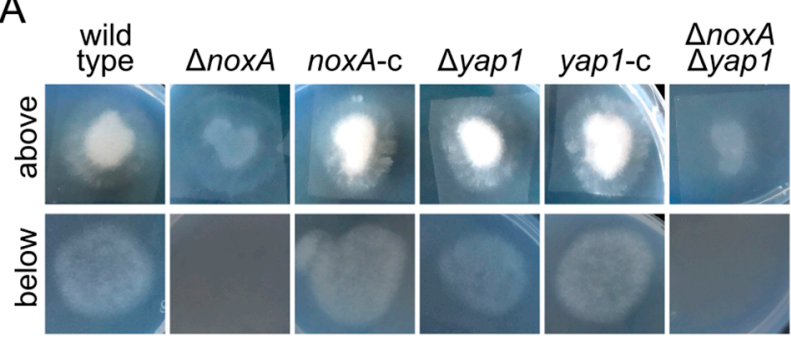

B

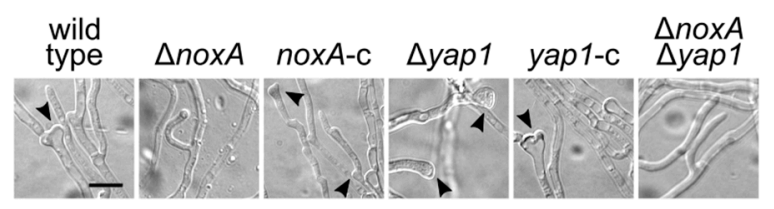

Figure 3. Penetration assays on cellophane membranes: (A) sheets of cellophane membrane were placed on CM agar, inoculated with conidial suspensions of the examined strains, and incubated for 5 days (above). Plates were scored for fungal growth 4 days after membrane removal (below). The experiments were performed in triplicate and 4 cellophane sheets per plate were used. (B) Microscopic examination of hyphopodial formation on the cellophane membranes (5 days postinoculation). Arrowheads: examples of hyphopodia. Bar $=5 \mu \mathrm{m}$.

\subsection{NoxA Is Involved in ROS Metabolism and Its Oxidative Activity Is Counteracted by Yap1}

Staining of $\mathrm{O}_{2}{ }^{\bullet-}$ radicals using NBT revealed their accumulation mostly in the apical parts of germlings and at the periphery of colonies of the $\Delta$ nox $A$ mutant, in contrast to their more uniform presence in the wild-type strain (Figure 4A). Peripheral staining of colonies was also observed in $\triangle$ nox $A \Delta y a p 1$, whereas the wild-type pattern was restored in the complemented strain (Figure 4A). These observations support the hypothesis that NoxA is involved in ROS metabolism in $V$. dahliae. On the other hand, deletion of yap1 resulted in generally increased formazan precipitation (Figure 4A), which indicates de- 
fects in ROS metabolism and is consistent with the conserved role of this regulator in ROS detoxification.

We further characterized the roles of NoxA and Yap1 in response to oxidative stress by exposing the wild-type and knockout strains to the oxidizing agent $\mathrm{H}_{2} \mathrm{O}_{2}$, the herbicide paraquat, the fungicide iprodione, and the ROS scavenger N-acetyl cysteine (NAC). As expected, we detected significant growth defects of $\triangle$ yap 1 in the presence of any ROSinducing agent (Figure $4 \mathrm{~B}, \mathrm{C}$ ). On the other hand, $\triangle$ noxA was inhibited to a lesser extent by $\mathrm{H}_{2} \mathrm{O}_{2}$ (at high concentrations), iprodione, and the ROS-scavenger NAC (Figure 4B,C). Notably, the double deletion mutant $\triangle$ noxA $\triangle$ yap 1 exhibited the same behavior as $\triangle$ nox $A$ (Figure 4B,C), indicating an epistatic relationship between the two genes.

A
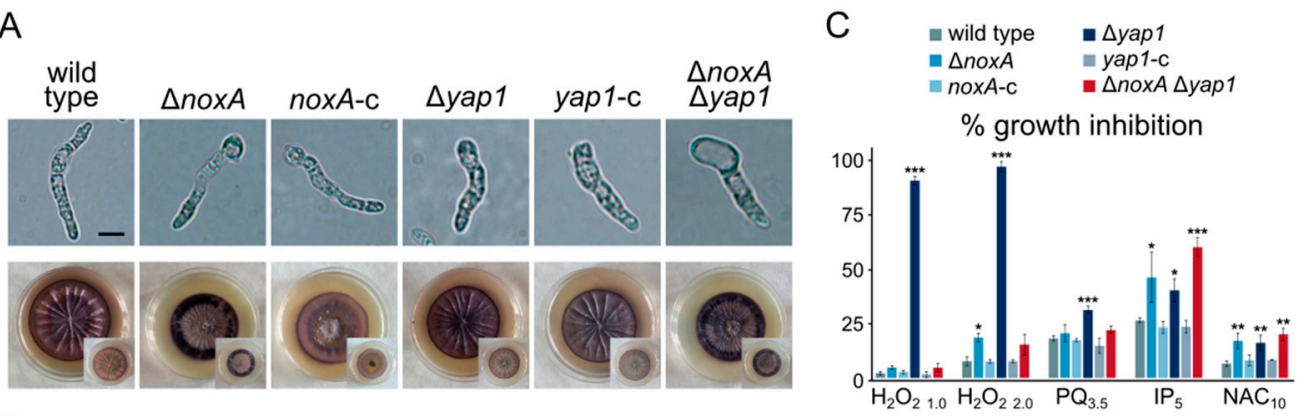

B

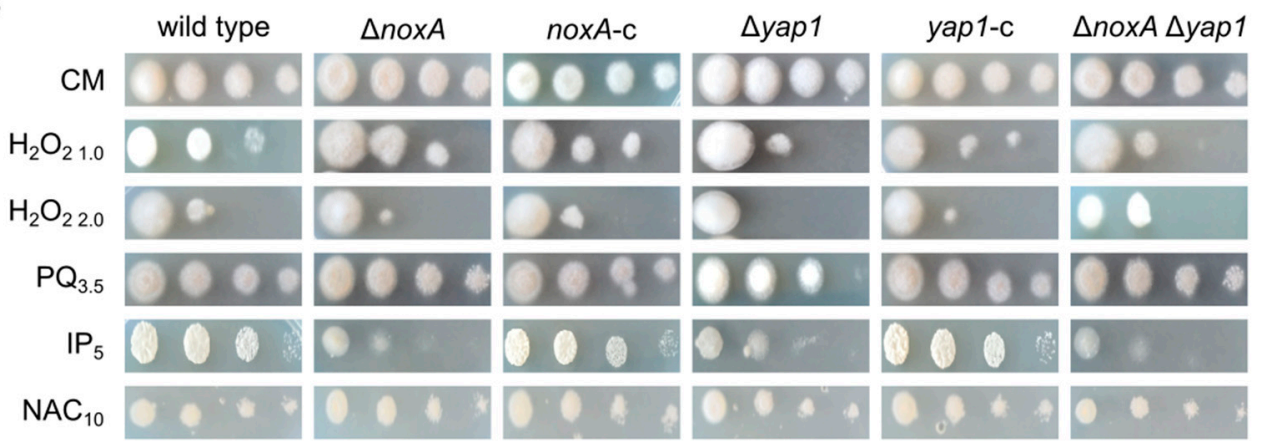

Figure 4. Patterns of ROS accumulation and oxidative stress tolerance of $V$. dahliae noxA and yap1 knockout mutants: (A) detection of $\mathrm{O}_{2}{ }^{\bullet-}$ using NBT staining in germlings (16 h of growth in CM) and colonies (20 days old on CM plates). Top and bottom views of plates are shown for each strain. (B) Conidial germination of each examined strain upon treatment with $\mathrm{H}_{2} \mathrm{O}_{2}$, paraquat, iprodione, or $\mathrm{N}$-acetyl cysteine (growth for 3 days). (C) Relative growth inhibition of colonies by the same substances. Bars: SD. Statistical significance of differences from the wild type was tested by Student's $t$-tests $\left({ }^{*} p \leq 0.05,{ }^{* *} p \leq 0.01,{ }^{* *} p \leq 0.001\right)$. Concentrations are expressed in $\mathrm{mM}$, except for iprodione $(\mu \mathrm{g} / \mathrm{mL})$. All experiments were performed in triplicate.

To gain a better understanding of the roles of $V$. dahliae nox $A$ and yap 1 in the OSR, we investigated their expression patterns before and after treatment with $\mathrm{H}_{2} \mathrm{O}_{2}$ by RT-qPCR (Figure 5). The noxA gene is strongly induced upon oxidative stress in the wild type, and neither its induction pattern nor its transcript levels were affected by deletion of yap1. On the other hand, yap1, which is down-regulated under oxidative stress in the wild type, failed to reach its normal expression levels in the $\triangle$ noxA mutant background under these conditions. When we also analyzed the expression levels of the stress-activated Hog1 MAP kinase gene before treatment with $\mathrm{H}_{2} \mathrm{O}_{2}$, we observed significantly lower levels in all knockout mutants than in the wild type. Under oxidative stress, however, hog 1 was down-regulated in the wild type, followed a similar but less pronounced trend in $\Delta y a p 1$, and moderate induction in $\triangle$ noxA and $\triangle$ noxA $\triangle$ yap 1 (Figure 5).

Furthermore, we characterized the responses to oxidative stress of three important downstream genes known to be involved in the OSR, i.e., catalase (cat1), superoxide dismutase (sod1), and glutathione reductase ( $g l r 1)$. As expected, induction of all three was detected in the wild-type strain in the presence of $\mathrm{H}_{2} \mathrm{O}_{2}$, whereas they were expressed 
at lower levels and failed to be induced by oxidative stress in the absence of their transcriptional regulator Yap1 (Figure 5). Interestingly, we detected significantly higher mRNA levels of cat 1 and $g l r 1$ in the $\triangle$ nox $A$ mutant than in the wild type under normal conditions, while sod 1 and $g l r 1$ failed to reach their wild-type levels under oxidative stress in $\Delta n o x A$ (Figure 5). The $\Delta$ noxA $\triangle y a p 1$ strain exhibited a similar expression pattern to $\Delta y a p 1$ for cat 1 and $g l r 1$, while the expression of $\operatorname{sod} 1$ was up-regulated under normal conditions in this strain (Figure 5). Our findings indicate that $V$. dahliae NoxA is up-regulated by oxidative stress and implicate it in the transcriptional regulation of both the OSR and the important stress-responsive MAP kinase Hog1.
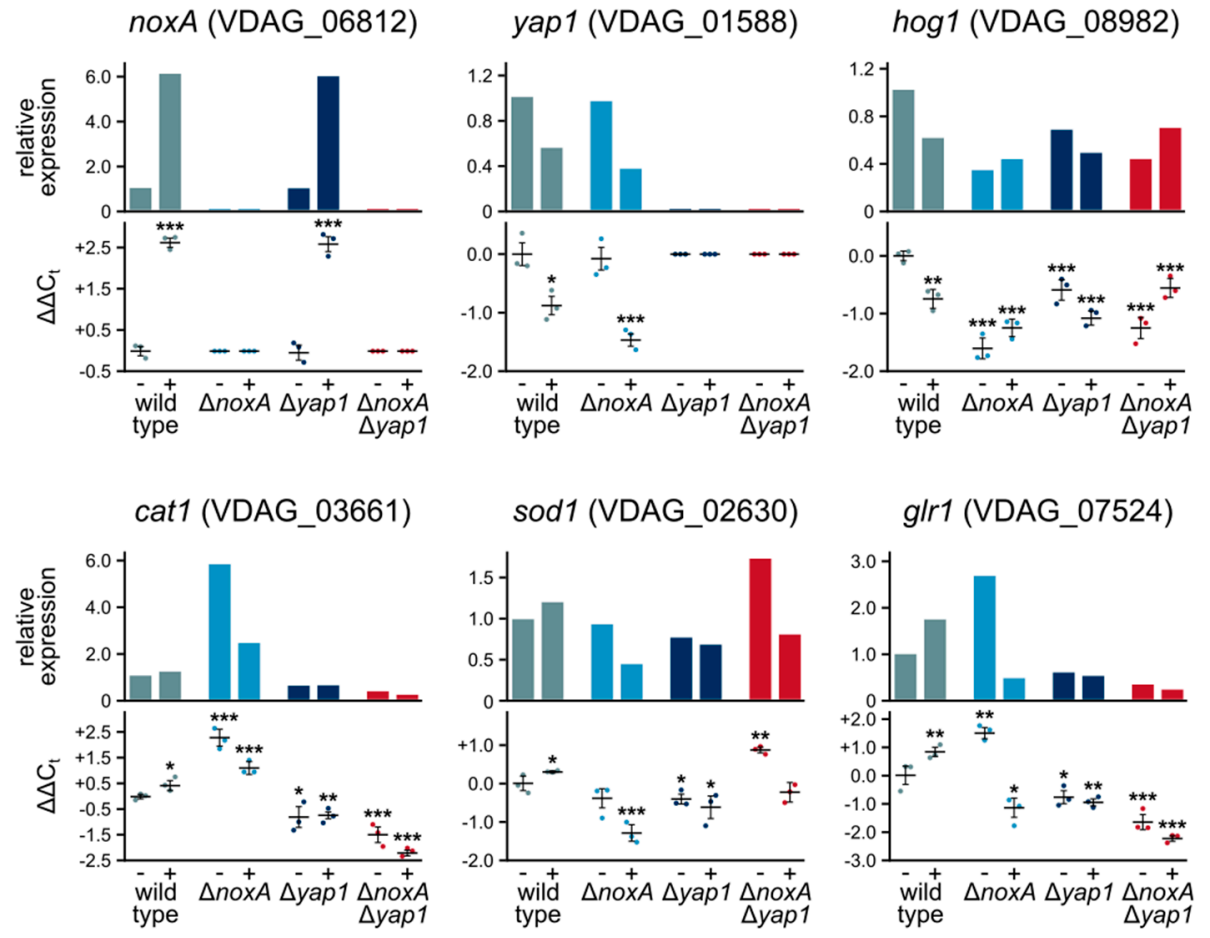

Figure 5. Expression profiles of genes involved in ROS metabolism before (-) and after (+) treatment with $1.5 \mathrm{mM} \mathrm{H}_{2} \mathrm{O}_{2}$. For each gene, the upper plot shows the average relative expression level in each strain compared to the untreated wild-type strain, and the lower shows the determined $\Delta \Delta \mathrm{Ct}$ values. Three biological replicates (and two technical replicates for each) were performed and analyzed for each strain and gene. In the $\Delta \Delta \mathrm{Ct}$ plots, the horizontal bars represent mean $\Delta \Delta \mathrm{Ct}$ values and the bars represent the SD between the three biological replicates. Statistical significance of differences from the untreated wild-type strain was tested by Student's $t$-tests $\left({ }^{*} p \leq 0.05,{ }^{* *} p \leq 0.01,{ }^{* * *} p \leq 0.001\right)$.

3.4. NoxA Is Involved in the Response to Cell Wall Stress and Possibly Interacts with Components of Important Biological Processes

We further investigated the possible involvement of NoxA and Yap1 in fungal responses to other sources of cellular stress by characterizing the behavior of the corresponding mutant strains in the presence of substances inducing hyperosmotic stress, cell wall-perturbating agents, high concentrations of trace metals, and various fungicides (Figure 6A,B). Regarding osmotic stress, only a minor growth inhibition of $\triangle$ noxA and $\Delta y a p 1$ was recorded in the presence of $\mathrm{NaCl}$. In contrast, cell wall stress significantly compromised the growth rates (calcofluor white and Congo red) and the germination frequency (calcofluor) of $\Delta n o x A$ and $\Delta n o x A \Delta y a p 1$, whereas $\Delta y a p 1$ exhibited limited sensitivity only to calcofluor. Consistently, the drug amphotericin B, which disorganizes fungal cell membranes by targeting ergosterol, and also causes oxidative stress, impaired growth and germination of $\triangle$ nox $A$ and $\triangle$ nox $A \Delta y a p 1$. However, the inhibitor of ergosterol biosynthesis fluconazole specifically restricted growth of $\Delta y a p 1$ and, to a lesser extent, that of the double deletion mutant. Motivated by the observation that deletion of yap 1 in yeast causes 
decreased resistance to metals [55], we checked resistance of the $V$. dahliae nox $A$ and yap1 mutants to $\mathrm{Ca}^{2+}, \mathrm{Cu}^{2+}$, and $\mathrm{Fe}^{2+}$ cations (Figure S3A,B, Supplementary Materials). While $\mathrm{Ca}^{2+}$ and $\mathrm{Cu}^{2+}$ indeed restricted radial growth of $\Delta y a p 1, \mathrm{Fe}^{2+}$ had a more pronounced effect on the noxA-deficient strains.

Apart from the Nox complexes, mitochondria are a major intracellular source of ROS. We tested fungicides that target the four complexes (I-IV) of the respiratory chain (i.e., azoxystrobin, sodium cyanide, pyraclostrobin, kresoxim-methyl, annisulborn, sodium amytal, flutolanil, and isopyrazam), and we found them all to cause significant growth reduction in $\triangle$ noxA and $\triangle$ noxA $\triangle$ yap1 strains, while $\triangle y a p 1$ was not affected (Figure $6 \mathrm{~A}, \mathrm{~B}$; Figure S3A,B, Supplementary Materials). These findings are suggestive of a possible crosstalk between the two major cellular sources of ROS.

Finally, since NoxA has been shown to interact with the machinery of autophagy in phagocytic cells [56], we tested the responses of the $V$. dahliae mutants to rapamycin, an inhibitor of the TORC1 kinase and, therefore, an inducer of autophagy (Figure 6A,B). Deletion of noxA, but not of yap1, led to significantly increased sensitivity to the inhibitor, which implies a possible dysregulation of autophagy.

A

\begin{tabular}{|c|c|c|c|c|c|c|}
\hline wild type & & $\Delta$ noxA & noxA-c & $\Delta$ уар1 & уар1-с & $\Delta \cap O X A \Delta y^{2}$ \\
\hline $\mathrm{cM} \bigcirc 000$ & & $\mathrm{OO}$ & 2000 & & 0000 & 000 \\
\hline $\mathrm{NaCl}_{1.0} \mathrm{O}$ & - & . & 00 & $0 \cdot$ & 0. & $\circ \cdot$ \\
\hline sorbitol $_{1.0} 0000$ & 0 & 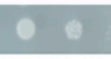 & 0000 & 000 & 90 & 000 \\
\hline calco. $50.0 \quad 0=$ & & & $\bullet$ & $0 A$ & $\mathbf{Q} \cdot$ & 0 \\
\hline Congo $_{30.0}$ & Q & 08 & 000 in & $000 *$ & 000 & $00 \%$ \\
\hline amph. B 0.1000 & 0 & 0 & 0008 & 000 & 000 & $00 \%$ \\
\hline flucon. $5.0 \mathrm{D}_{4}$ & 6 & $\cdot \cdot$ & $2+2$ & + & $8 x$ & \\
\hline azoxys. 5.0 & 0 & $\circ$ & 00 & $0 \circ$ & 0. & 000 \\
\hline yanide $_{400.0}$ & O & $0 *$ & 0.0 & $00 \%$ & $00^{\circ}$ & $00^{\circ}$ \\
\hline raclos. 10.000 & 0 & 0 & 000 & 00 & 000 & $00=$ \\
\hline esoxim $_{50.0}$ & 0 & + & $0 *$ & $0 *$ & 8. & $08+4$ \\
\hline rapam. $50.0 \mathrm{O}$, & 0 & + & $0+4$ & $0 *$ & e. & $0 \times$ \\
\hline
\end{tabular}

B $\square$ wild type $\square \Delta$ noxA $\square$ noxA-c $\square \Delta$ yap1 $\square$ yap1-c $\square \Delta$ noxA $\Delta y a p 1$

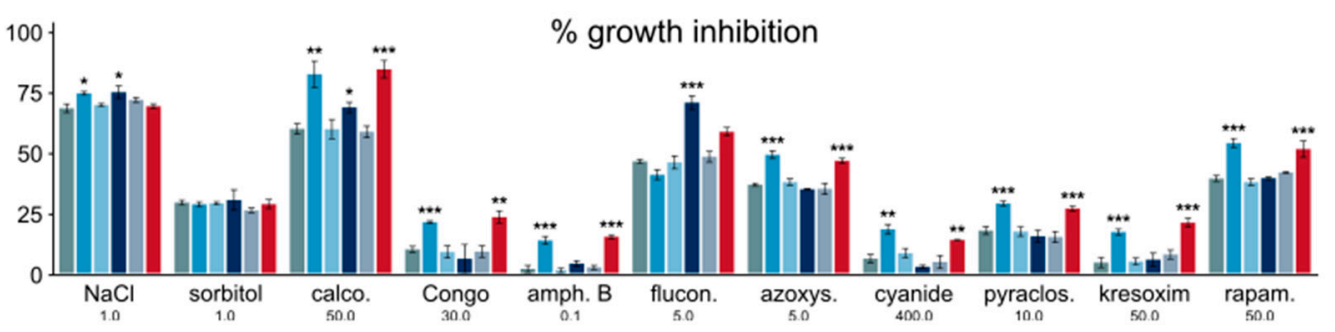

Figure 6. Effects of osmotic stress, cell wall- and plasma membrane-perturbating agents, and antifungal agents that target the respiratory chain on $V$. dahliae $\triangle$ nox $A$ and $\triangle y a p 1$ mutants: (A) effects of $\mathrm{NaCl}$, sorbitol, calcofluor white M2R, Congo red, amphotericin B, fluconazole, azoxystrobin, sodium cyanide, pyraclostrobin, kresoxim-methyl, and rapamycin on conidial germination (growth for 3 days). (B) Relative growth inhibition of colonies caused by the same substances. Bars: SD. Statistical significance of differences from the wild type was tested by Student's $t$-tests $\left({ }^{*} p \leq 0.05\right.$, $\left.{ }^{* *} p \leq 0.01,{ }^{* * *} p \leq 0.001\right)$. All concentrations are expressed in $\mu \mathrm{g} / \mathrm{mL}$, except for $\mathrm{NaCl}(\mathrm{M})$, sorbitol $(\mathrm{M})$, and rapamycin $(\mathrm{nM})$. All experiments were performed in triplicate. 


\subsection{NoxA, but Not Yap1, Is Essential in Both Partners for CAT-Mediated Cell Fusion}

We have previously found that NoxA in $V$. dahliae is essential for somatic fusion of conidia or germlings via CATs [23]. On the contrary, deletion of yap1 exerted no effect on CAT-mediated fusion (Figure 7A). The double deletion mutant exhibited the same behavior as $\triangle$ noxA (Figure 7A). Surprisingly, the $\triangle$ noxA mutant retained its capacity for hyphal fusion between mature hyphae (Figure 7B), which suggests differences between the regulatory mechanisms of conidial and hyphal fusion.

A

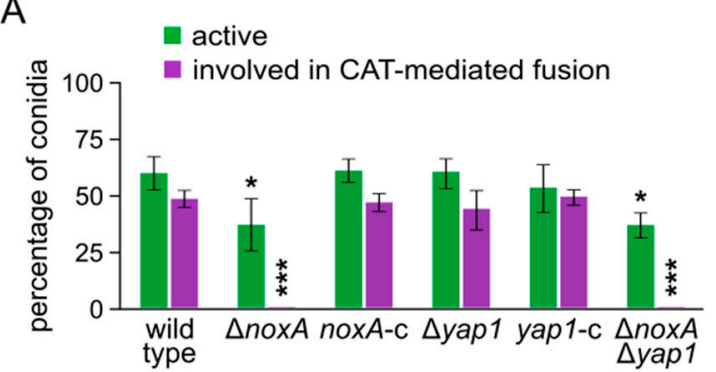

C

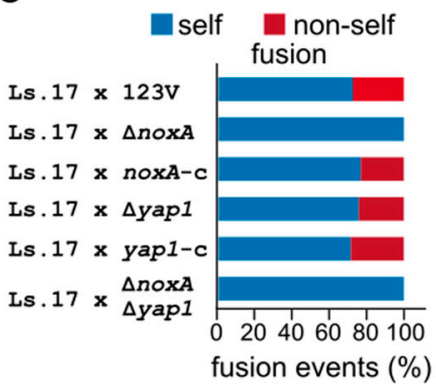

$\mathrm{D}$

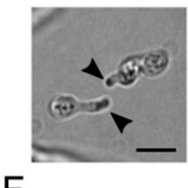

F
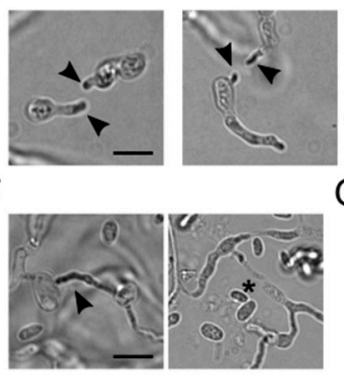

B

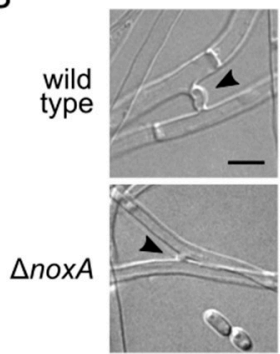

$\mathrm{E}$

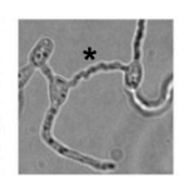

G - inviable fusions

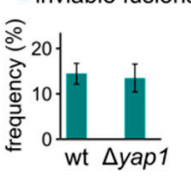

$\triangle$ yap1
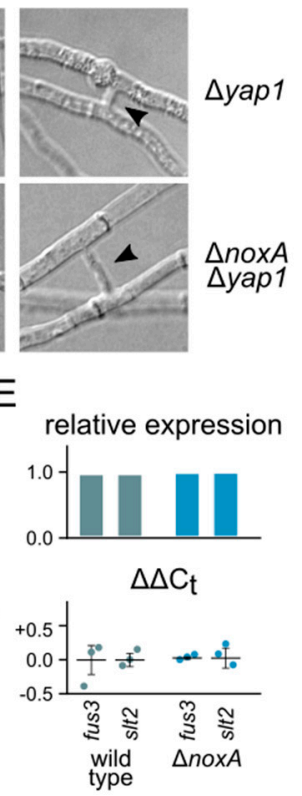

Figure 7. Roles of $V$. dahliae NoxA and Yap1 in somatic cell fusion and the heterokaryon incompatibility reaction: (A) competence of deletion mutants in CAT-mediated fusion of conidia or germlings. Frequencies of active conidia and their fraction that are involved in CAT fusion are shown for each strain. All assays were performed in triplicate and 150 conidia were analyzed per replicate. Bars: SD. Statistical significance of differences from the wild type was tested by Student's $t$-tests $\left(^{*} p \leq 0.05,{ }^{* * *}\right.$ $p \leq 0.001$ ). (B) Fusions between mature hyphae of the indicated strains. (C) Frequencies of self and nonself fusions in pairings of the indicated strains with their incompatible partner, Ls.17 H1-sgfp. Each pairing was tested in triplicate, and 150 fusion events were analyzed per replicate. (D) Detection of $\mathrm{O}_{2}{ }^{\bullet-}$ by NBT staining during CAT homing and establishment of CAT fusion (wild-type strain $123 \mathrm{~V})$. Arrowheads: localized ROS production at CAT tips during homing; asterisk: a complete CAT fusion. Bar $=5 \mu \mathrm{m}$. (E) Relative expression levels of the MAPK genes fus 3 and slt2 in the wild-type and $\Delta$ noxA strains. The upper plot shows the average relative expression levels compared to the corresponding genes of the wild type, and the lower shows the determined $\Delta \Delta \mathrm{Ct}$ values. Three biological replicates (and two technical replicates for each) were analyzed for each strain and gene. In the $\Delta \Delta \mathrm{Ct}$ plots, the horizontal bars represent mean $\Delta \Delta \mathrm{Ct}$ values, and the bars $\mathrm{SD}$ between the three biological replicates. Statistical significance of differences from the untreated wild-type strain was tested by Student's $t$-tests $\left({ }^{* *} p \leq 0.01,{ }^{* * *} p \leq 0.001\right.$ ). (F) Detection of $\mathrm{O}_{2}{ }^{\bullet-}$ by NBT staining (arrowhead) during the heterokaryon incompatibility reaction $(123 \mathrm{~V} \times$ Ls.17 pairing). Inviable cells were no longer stained with NBT (asterisk). (G) Frequency of inviable fusions determined by staining with methylene blue in $123 \mathrm{~V} \times$ Ls.17 and $123 \mathrm{~V} \Delta$ yap $1 \times$ Ls.17 pairings. Each pairing was tested in triplicate $(n=100$ anastomoses per replicate). Bar $=5 \mu \mathrm{m}$.

We hypothesized that the role of NoxA in cell fusion via CATs could be related to the generation or perception of the unknown signal that is involved in pre-fusion cell communication. If that process were unidirectional (i.e., each fusion partner emitting or receiving the signal, but not both), a functional copy of nox $A$ in only one of the interacting partners could be sufficient for fusion of a subset of cells. To test this possibility, we paired the deletion 
strains with the strain Ls.17 H1-sgfp (expressing GFP-tagged histone H1 in its nuclei), which permitted the microscopic identification of nonself fusions. The presence of functional nox $A$ genes in both partners was found to be essential for their fusion (Figure 7C); deletion of yap1 from one partner showed no effect, as expected.

It is unclear whether NoxA functions in cell fusion by causing localized ROS production with direct effects on partner recognition or the commitment to fuse, or indirectly by transducing signals to alter the expression of other genes or the activity of their products. We found that, during the homing phase of the interacting cells prior to their fusion, $\mathrm{O}_{2}{ }^{\bullet-}$ always accumulated at the tips of the mutually attracted CATs (Figure 7D), suggesting that localized generation of ROS putatively by NoxA is indeed involved in the process. No ROS accumulation was detected after completion of CAT-mediated fusion (Figure 7D). On the other hand, deletion of nox $A$ had no effect on the expression levels of the MAP kinases Fus3 and Slt2, which are important components of the pre-fusion communication mechanism in N. crassa [22], and are also required for CAT fusion in V. dahliae [23].

Fusion of vegetatively incompatible strains of fungi, including $V$. dahliae, usually triggers an incompatibility reaction that can cause cell death of the anastomosed compartments [54]. Based on the observations that this reaction in N. crassa is characterized by an induction of ROS [57], and that genes involved in the OSR are up-regulated during CAT formation in Colletotrichum gloeosporioides [58], we used NBT staining to study $\mathrm{O}_{2}{ }^{\bullet-}$ accumulation in incompatible $V$. dahliae fusions (pairing Ls.17 H1-sgfp $\times 123 \mathrm{~V}$ ). One-third of such fusions $(n=30)$ exhibited increased formazan precipitation (Figure 7F), indicating that ROS accumulation is indeed associated with the onset of the incompatibility reaction, similarly to what was observed in N. crassa [57]. Deletion of yap1 had no effect on the frequency of inviable fusions (Ls.17 H1-sgfp $\times 123 \mathrm{~V} \Delta$ yap1 pairing), which suggests either that the observed ROS accumulation does not mediate the catastrophic reaction or that Yap1 does not respond to it.

\section{Discussion}

The dual nature of ROS in biological systems as a potential cause of cellular damage on the one hand, and as signaling components of important developmental processes on the other, has been the focus of extensive research $[1,2,5,8]$. This duality is clearly illustrated in the case of fungal plant pathogens, which need to cope with their host-derived ROS bursts during infection [4], while they generate and use endogenous ROS for their own development and pathogenicity [5,6]. Therefore, the survival and ecological success of these organisms depend on their ability to maintain a redox balance that presumably requires coordination of ROS generation and scavenging systems. In this study, we investigated the roles of the ROS-producing NADPH oxidase NoxA in the biology of the important plantpathogenic fungus $V$. dahliae, in comparison to its transcription factor Yap1, a conserved central regulator of ROS detoxification $[13,28]$. We found that NoxA has multifaceted roles in important developmental and physiological processes, plays an essential role in virulence, mediates responses to different types of cellular stress, and is required for somatic cell fusion.

Fungal NADPH oxidase complexes have been attributed pleiotropic functions in morphogenesis and pathogenicity $[9,12,13,59]$. In $V$. dahliae, we found NoxA to be required for normal formation of microsclerotia, i.e., dormant resting structures that are crucial for long-term survival and spread of the species [42]. In addition, $V$. dahliae NoxA is necessary for normal formation of aerial hyphae and germination of its asexual spores, in agreement with similar previously described roles in, e.g., N. crassa, Epichloë festucae, C. purpurea, and S. macrospora [16-18,60]. Furthermore, our investigation revealed that NoxA is essential for virulence in $V$. dahliae, and our findings suggest that this can be at least partly due to its involvement in the formation of hyphopodia, i.e., infectious swollen hyphae that can penetrate the roots of the plant hosts to initiate the disease cycle $[43,44]$. Although the regulatory mechanisms that underlie the development of $V$. dahliae hyphopodia are mostly unknown, recent reports implicated in this process both Ste11, a component of MAP 
kinase signaling, and Csin1, a cellophane surface-induced protein that acts through the cAMP pathway $[61,62]$. These findings suggest putative interactions of ROS with important signaling transduction pathways. Previous studies have demonstrated the involvement of NoxA in the formation of appressoria in the plant pathogen M. oryzae $[10,11]$ and traps in the nematode pathogen Arthrobotrys oligospora [63]. These findings together indicate conserved involvement of the ROS-generating enzyme NoxA in the early stages of infection of diverse pathogenic fungi.

Another explanation for the highly compromised ability of $V$. dahliae to cause systemic infection and disease symptoms in the absence of NoxA could be its increased sensitivity to various types of environmental stress, most notably cell wall and oxidative stress. Oxidative bursts of ROS have been described to play a role in cell wall biosynthesis [9], which could be linked to the sensitivity of the $V$. dahliae $\Delta$ nox $A$ mutant to cell wall-perturbating agents. Our analyses also revealed reduced resistance of $\triangle$ nox $A$ to oxidative stress, which could be attributed to dysregulation of antioxidant genes, such as the example of sod1 that we observed in our study. In addition, deletion of nox $A$ resulted in dysregulation of the important stress-activated MAP kinase Hog1, which could be directly or indirectly linked to some of the identified pleiotropic phenotypes of NoxA. These observations could be likely attributed to the expected function of ROS as secondary signals that could potentially control the expression and function of multiple transcription factors and signaling components via post-translational modification [2]. A possible mechanism for this could involve the control of cellular localization of key signaling components, such as the MAP kinase Mpk1 in P. anserina, whose normal localization depends on nox1 [64]. Notably, the absence of functional NoxA rendered the fungus sensitive to several antifungal agents, including representatives that block the central TOR kinase, as well as the four complexes of the mitochondrial electron transport chain. The latter observation indicates that the maintenance of a minimum level of intracellular ROS is important for cell functionality and viability. It could also be relevant to the crosstalk that has been demonstrated between mitochondrial ROS and NADPH oxidases in mammalian cells [65], hypothetically reflecting the function of mechanisms that ensure the coordination of those two major intracellular sources of ROS in the context of oxidative homeostasis.

Conidial and hyphal fusion is an integral component of the establishment and development of fungal colonies [66], and nonself fusion could possibly grant access to parasexual generation of diversity [23,54]. We have previously demonstrated that NoxA is essential for CAT-mediated fusion of $V$. dahliae conidia or germlings [23], and we further found in the present study that the requirement for functional NoxA applies to both interacting cells. We also detected $\mathrm{O}_{2}{ }^{\bullet-}$ accumulation at the tips of wild-type germlings during their homing phase of interaction prior to fusion, which could most likely be attributed to the activity of NoxA. Similar observations have previously been made in B. cinerea [25]. We hypothesize that temporally and spatially regulated ROS bursts could be involved in the pre-fusion communication of the interacting cells, possibly linked to the oscillatory recruitment of signaling components (such as the MAP kinase Fus3 and the scaffold protein SOFT) to cell tips that controls cell fusion in N. crassa [67].

The transcription factor Yap1 has a conserved role in ROS detoxification among diverse organisms as a central regulatory element that participates in the orchestration of their OSR [30-37]. Indeed, we found deletion of yap1 to result in increased sensitivity to all oxidative agents tested, as well as to the fungicide fluconazole, which can induce ROS production in Cryptococcus neoformans [68,69]. In addition, the expression of important antioxidant genes, such as cat1, sod1, and $g l r 1$, was lower and non-inducible by oxidative stress in the absence of Yap1. Apart from its regulatory function in oxidative stress tolerance, Yap1 has also been attributed additional developmental and other, sometimes contradictory, roles between fungi [28]. Here, we found that in $V$. dahliae Yap1 is necessary for normal conidiation, in line with previous findings in M. oryzae and A. nidulans [33,37], while its deletion also severely compromised the formation of microsclerotia. A previous study demonstrated attenuated microsclerotial formation upon $V$. dahliae yap1 deletion [40], to 
a lesser extent than the one observed in our work, which probably reflects strain-specific variation in the fine-tuning of the underlying regulatory mechanisms. In agreement with the previous study that analyzed virulence on smoke trees [40], we detected no significant involvement of Yap1 in the virulence of $V$. dahliae on eggplant. This is consistent with studies of other fungal pathogens that do not require Yap1 for infecting their hosts (e.g., B. cinerea, Fusarium graminearum, and Cochliobolus heterostrophus), but in contrast to other fungi where Yap1 is indispensable for successful infection (e.g., Ustilago maydis, M. oryzae, and Alternaria alternata) $[31,33,35,38,70,71]$. These findings suggest that, in some fungi, alternative detoxification pathways or regulatory mechanisms likely take over during infection and suffice for efficient ROS detoxification when Yap1 is absent. Such alternatives could include the transcription factors Atf1 and Skn7, which mediate responses to RNS and ROS, respectively, by regulating the expression of antioxidant genes [72,73].

Based on the significant biological roles of the ROS-generating NADPH oxidase NoxA in $V$. dahliae, and the presumed need of the pathogen to withstand the ROS bursts involved in the defense mechanisms of its hosts [4], we would expect systems for coordination between ROS generation and processing to be important for its ROS homeostasis. In support of this hypothesis, our study revealed an antagonistic epistatic relationship between noxA and yap1, with their double null mutant exhibiting the same behavior as $\triangle$ noxA in all tested phenotypes, instead of any detectable additivity between the two genes. This was also observed with regard to sensitivity to oxidative stress, whereby $\triangle$ nox $A$ and $\triangle$ nox $A$ $\triangle$ yap 1 were more resistant to oxidative agents than $\triangle$ yap1. Our interpretation of these findings is that Yap1 contributes to the neutralization of the ROS produced by NoxA, but this Yap1 detoxification function depends on NoxA, which itself responds to oxidative stress with higher expression levels. We propose that, in the absence of NoxA, other yap1independent systems take over, presumably via up-regulation, to effectively shield the organism from the hazards of elevated oxidative stress. This is further supported by the detected changes in the expression patterns of antioxidant genes in the $\triangle$ noxA mutant. The observed small increase in Hog1 transcripts observed in this strain is unlikely to mediate antioxidant responses, since, in V. dahliae, Hog1 appears to play no role in the OSR [74]. In other fungi, oxidative stress signaling alternatives include Pap1 activation by Tpx1 and the Hog1 homologs Sty1/Spc1 MAPK pathway, which can trigger antioxidant responses in Schizosaccharomyces pombe [75]. Furthermore, the OSR of the filamentous fungus $A$. nidulans involves, in addition to its Yap1 homolog NapA, the Skn7 homolog SrrA and the Hog1 homologs SakA and MpkC [76-78]. Finally, we would attribute the findings that low levels of oxidative stress (e.g., low concentrations of $\mathrm{H}_{2} \mathrm{O}_{2}$ ) have no observable effect on the $\triangle$ noxA strain and, consistently, no induction of antioxidant genes, to the perturbated generation of endogenous ROS, which could be linked to a higher resistance of the fungus to low levels of exogenous oxidative stress.

In this study, we characterized the $V$. dahliae homolog of the important ROS producer NoxA, and we demonstrated significant pleiotropic roles in multiple developmental and physiological processes. We provide evidence that implicates NoxA in sensing and mediating responses to oxidative stress through genetic interactions with the transcriptional regulator Yap1 and presumably alternative, currently unknown, ROS signaling and detoxification systems. These data contribute to a better understanding of fungal ROS homeostasis and welcome future research to elucidate the significance and modes of action of ROS metabolism in the development, pathogenicity, and stress response of fungal pathogens.

Supplementary Materials: The following are available online at https:/ / www.mdpi.com/article/10 .3390/jof7090740/s1, Figure S1: Sequence alignment of Yap1 homologs of V. dahliae (VDAG_01588) and other fungi, Figure S2: Validation of the yap1 knockout mutants, Figure S3: Effects of antifungal agents that target the respiratory chain, and trace elements on $V$. dahliae deletion mutants $\triangle$ nox $A$ and $\Delta$ yap1, Table S1: Verticillium dahliae strains constructed and used in this study, Table S2: List of plasmids constructed and used in this study, Table S3: List of DNA oligonucleotides used in this study. 
Author Contributions: Conceptualization, I.A.P., V.V. and M.A.T.; methodology, I.A.P., V.V., E.A.M. and M.A.T.; formal analysis, V.V. and E.A.M.; investigation, V.V., I.A.P. and E.A.M.; resources, M.A.T. and M.K.; visualization, V.V.; writing-original draft preparation, V.V and I.A.P.; writing-review and editing, I.A.P., M.A.T., V.V. and E.A.M.; supervision, M.A.T. and I.A.P.; funding acquisition, M.A.T. and M.K. All authors have read and agreed to the published version of the manuscript.

Funding: This research is co-financed by Greece and the European Union (European Social FundESF) through the Operational Programme «Human Resources Development, Education and Lifelong Learning» in the context of the project "Strengthening Human Resources Research Potential via Doctorate Research" (MIS-5000432), implemented by the State Scholarships Foundation (IK $\Upsilon$ ).

Institutional Review Board Statement: Not applicable.

Informed Consent Statement: Not applicable.

Data Availability Statement: The data that support the findings of this study are available in the main text, figures, and additional files of this article.

Acknowledgments: We are grateful to E. K. Ligoxigakis (NAGREF-DEMETER, Heraklion, Greece) for his valuable help with the pathogenicity bioassay.

Conflicts of Interest: The authors declare no conflict of interest.

\section{References}

1. Lambeth, J.D.; Neish, A.S. Nox Enzymes and New Thinking on Reactive Oxygen: A Double-Edged Sword Revisited. Annu. Rev. Pathol. Mech. Dis. 2014, 9, 119-145. [CrossRef]

2. Sies, H.; Jones, D.P. Reactive Oxygen Species (ROS) as Pleiotropic Physiological Signalling Agents. Nat. Rev. Mol. Cell Biol. 2020, 21, 363-383. [CrossRef]

3. Finkel, T. Oxygen Radicals and Signaling. Curr. Opin. Cell Biol. 1998, 10, 248-253. [CrossRef]

4. Doehlemann, G.; Hemetsberger, C. Apoplastic Immunity and Its Suppression by Filamentous Plant Pathogens. New Phytol. 2013, 198, 1001-1016. [CrossRef] [PubMed]

5. Heller, J.; Tudzynski, P. Reactive Oxygen Species in Phytopathogenic Fungi: Signaling, Development, and Disease. Annu. Rev. Phytopathol. 2011, 49, 369-390. [CrossRef]

6. Zhang, Z.; Chen, Y.; Li, B.; Chen, T.; Tian, S. Reactive Oxygen Species: A Generalist in Regulating Development and Pathogenicity of Phytopathogenic Fungi. Comput. Struct. Biotechnol. J. 2020, 18, 3344-3349. [CrossRef]

7. Murphy, M.P. How Mitochondria Produce Reactive Oxygen Species. Biochem. J. 2009, 417, 1-13. [CrossRef] [PubMed]

8. Lambeth, J.D. NOX Enzymes and the Biology of Reactive Oxygen. Nat. Rev. Immunol. 2004, 4, 181-189. [CrossRef] [PubMed]

9. Takemoto, D.; Tanaka, A.; Scott, B. NADPH Oxidases in Fungi: Diverse Roles of Reactive Oxygen Species in Fungal Cellular Differentiation. Fungal Genet. Biol. 2007, 44, 1065-1076. [CrossRef] [PubMed]

10. Egan, M.J.; Wang, Z.-Y.; Jones, M.A.; Smirnoff, N.; Talbot, N.J. Generation of Reactive Oxygen Species by Fungal NADPH Oxidases Is Required for Rice Blast Disease. Proc. Natl. Acad. Sci. USA 2007, 104, 11772-11777. [CrossRef] [PubMed]

11. Ryder, L.S.; Dagdas, Y.F.; Mentlak, T.A.; Kershaw, M.J.; Thornton, C.R.; Schuster, M.; Chen, J.; Wang, Z.; Talbot, N.J. NADPH Oxidases Regulate Septin-Mediated Cytoskeletal Remodeling during Plant Infection by the Rice Blast Fungus. Proc. Natl. Acad. Sci. USA 2013, 110, 3179-3184. [CrossRef]

12. Segal, L.M.; Wilson, R.A. Reactive Oxygen Species Metabolism and Plant-Fungal Interactions. Fungal Genet. Biol. 2018, 110, 1-9. [CrossRef]

13. Scott, B. Conservation of Fungal and Animal Nicotinamide Adenine Dinucleotide Phosphate Oxidase Complexes. Mol. Microbiol. 2015, 95, 910-913. [CrossRef] [PubMed]

14. Lara-Ortíz, T.; Riveros-Rosas, H.; Aguirre, J. Reactive Oxygen Species Generated by Microbial NADPH Oxidase NoxA Regulate Sexual Development in Aspergillus Nidulans. Mol. Microbiol. 2003, 50, 1241-1255. [CrossRef] [PubMed]

15. Malagnac, F.; Lalucque, H.; Lepère, G.; Silar, P. Two NADPH Oxidase Isoforms Are Required for Sexual Reproduction and Ascospore Germination in the Filamentous Fungus Podospora Anserina. Fungal Genet. Biol. 2004, 41, 982-997. [CrossRef]

16. Dirschnabel, D.E.; Nowrousian, M.; Cano-Domínguez, N.; Aguirre, J.; Teichert, I.; Kück, U. New Insights Into the Roles of NADPH Oxidases in Sexual Development and Ascospore Germination in Sordaria Macrospora. Genetics 2014, 196, 729-744. [CrossRef]

17. Cano-Domínguez, N.; Álvarez-Delfín, K.; Hansberg, W.; Aguirre, J. NADPH Oxidases NOX-1 and NOX-2 Require the Regulatory Subunit NOR-1 To Control Cell Differentiation and Growth in Neurospora Crassa. Eukaryot. Cell 2008, 7, 1352-1361. [CrossRef]

18. Giesbert, S.; Schürg, T.; Scheele, S.; Tudzynski, P. The NADPH Oxidase Cpnox1 Is Required for Full Pathogenicity of the Ergot Fungus Claviceps Purpurea. Mol. Plant Pathol. 2008, 9, 317-327. [CrossRef] [PubMed]

19. Kim, H.J. Exploitation of Reactive Oxygen Species by Fungi: Roles in Host-Fungus Interaction and Fungal Development. J. Microbiol. Biotechnol. 2014, 24, 1455-1463. [CrossRef] 
20. Segmüller, N.; Kokkelink, L.; Giesbert, S.; Odinius, D.; van Kan, J.; Tudzynski, P. NADPH Oxidases Are Involved in Differentiation and Pathogenicity in Botrytis Cinerea. Mol. Plant-Microbe Interact. 2008, 21, 808-819. [CrossRef]

21. Yang, S.L.; Chung, K.-R. The NADPH Oxidase-Mediated Production of Hydrogen Peroxide (H2O2) and Resistance to Oxidative Stress in the Necrotrophic Pathogen Alternaria Alternata of Citrus. Mol. Plant Pathol. 2012, 13, 900-914. [CrossRef] [PubMed]

22. Fischer, M.S.; Glass, N.L. Communicate and Fuse: How Filamentous Fungi Establish and Maintain an Interconnected Mycelial Network. Front. Microbiol. 2019, 10, 619. [CrossRef]

23. Vangalis, V.; Knop, M.; Typas, M.A.; Papaioannou, I.A. Establishment of Conidial Fusion in the Asexual Fungus Verticillium Dahliae as a Useful System for the Study of Non-Sexual Genetic Interactions. Curr. Genet. 2021, 67, 471-485. [CrossRef] [PubMed]

24. Nordzieke, D.E.; Fernandes, T.R.; Ghalid, M.E.; Turrà, D.; Pietro, A.D. NADPH Oxidase Regulates Chemotropic Growth of the Fungal Pathogen Fusarium Oxysporum towards the Host Plant. New Phytol. 2019, 224, 1600-1612. [CrossRef]

25. Roca, M.G.; Weichert, M.; Siegmund, U.; Tudzynski, P.; Fleissner, A. Germling Fusion via Conidial Anastomosis Tubes in the Grey Mould Botrytis Cinerea Requires NADPH Oxidase Activity. Fungal Biol. 2012, 116, 379-387. [CrossRef]

26. Shelest, E. Transcription Factors in Fungi. FEMS Microbiol. Lett. 2008, 286, 145-151. [CrossRef]

27. Jakoby, M.; Weisshaar, B.; Dröge-Laser, W.; Vicente-Carbajosa, J.; Tiedemann, J.; Kroj, T.; Parcy, F. BZIP Transcription Factors in Arabidopsis. Trends Plant Sci. 2002, 7, 106-111. [CrossRef]

28. Mendoza-Martínez, A.E.; Cano-Domínguez, N.; Aguirre, J. Yap1 Homologs Mediate More than the Redox Regulation of the Antioxidant Response in Filamentous Fungi. Fungal Biol. 2020, 124, 253-262. [CrossRef]

29. Delaunay, A.; Isnard, A.-D.; Toledano, M.B. $\mathrm{H}_{2} \mathrm{O}_{2}$ Sensing through Oxidation of the Yap1 Transcription Factor. EMBO J. 2000, 19, 5157-5166. [CrossRef] [PubMed]

30. Lessing, F.; Kniemeyer, O.; Wozniok, I.; Loeffler, J.; Kurzai, O.; Haertl, A.; Brakhage, A.A. The Aspergillus Fumigatus Transcriptional Regulator AfYap1 Represents the Major Regulator for Defense against Reactive Oxygen Intermediates but Is Dispensable for Pathogenicity in an Intranasal Mouse Infection Model. Eukaryot. Cell 2007, 6, 2290-2302. [CrossRef]

31. Temme, N.; Tudzynski, P. Does Botrytis Cinerea Ignore $\mathrm{H}(2) \mathrm{O}(2)$-Induced Oxidative Stress during Infection? Characterization of Botrytis Activator Protein 1. Mol. Plant Microbe Interact. 2009, 22, 987-998. [CrossRef] [PubMed]

32. Takahashi, M.; Yamashita, K.; Shiozawa, A.; Ichiishi, A.; Fukumori, F.; Fujimura, M. An AP-1-like Transcription Factor, NAP-1, Regulates Expression of the Glutathione S-Transferase and NADH:Flavin Oxidoreductase Genes in Neurospora Crassa. Biosci. Biotechnol. Biochem. 2010, 74, 746-752. [CrossRef]

33. Guo, M.; Chen, Y.; Du, Y.; Dong, Y.; Guo, W.; Zhai, S.; Zhang, H.; Dong, S.; Zhang, Z.; Wang, Y.; et al. The BZIP Transcription Factor MoAP1 Mediates the Oxidative Stress Response and Is Critical for Pathogenicity of the Rice Blast Fungus Magnaporthe Oryzae. PLoS Pathog. 2011, 7, e1001302. [CrossRef] [PubMed]

34. Cartwright, G.M.; Scott, B. Redox Regulation of an AP-1-Like Transcription Factor, YapA, in the Fungal Symbiont Epichloë Festucae. Eukaryot. Cell 2013, 12, 1335-1348. [CrossRef]

35. Montibus, M.; Ducos, C.; Bonnin-Verdal, M.-N.; Bormann, J.; Ponts, N.; Richard-Forget, F.; Barreau, C. The BZIP Transcription Factor Fgap1 Mediates Oxidative Stress Response and Trichothecene Biosynthesis but Not Virulence in Fusarium Graminearum. PLoS ONE 2013, 8, e83377. [CrossRef] [PubMed]

36. Li, X.; Wu, Y.; Liu, Z.; Zhang, C. The Function and Transcriptome Analysis of a BZIP Transcription Factor CgAP1 in Colletotrichum Gloeosporioides. Microbiol. Res. 2017, 197, 39-48. [CrossRef]

37. Mendoza-Martínez, A.E.; Lara-Rojas, F.; Sánchez, O.; Aguirre, J. NapA Mediates a Redox Regulation of the Antioxidant Response, Carbon Utilization and Development in Aspergillus Nidulans. Front. Microbiol. 2017, 8, 516. [CrossRef]

38. Molina, L.; Kahmann, R. An Ustilago Maydis Gene Involved in $\mathrm{H}_{2} \mathrm{O}_{2}$ Detoxification Is Required for Virulence. Plant Cell 2007, 19, 2293-2309. [CrossRef]

39. Dankai, W.; Pongpom, M.; Youngchim, S.; Cooper, C.R.; Vanittanakom, N. The YapA Encodes BZIP Transcription Factor Involved in Stress Tolerance in Pathogenic Fungus Talaromyces Marneffei. PLoS ONE 2016, 11, e0163778. [CrossRef]

40. Tang, C.; Jin, X.; Klosterman, S.J.; Wang, Y. Convergent and Distinctive Functions of Transcription Factors VdYap1, VdAtf1, and VdSkn7 in the Regulation of Nitrosative Stress Resistance, Microsclerotia Formation, and Virulence in Verticillium Dahliae. Mol. Plant Pathol. 2020, 21, 1451-1466. [CrossRef]

41. Reverberi, M.; Zjalic, S.; Ricelli, A.; Punelli, F.; Camera, E.; Fabbri, C.; Picardo, M.; Fanelli, C.; Fabbri, A.A. Modulation of Antioxidant Defense in Aspergillus Parasiticus Is Involved in Aflatoxin Biosynthesis: A Role for the ApyapA Gene. Eukaryot. Cell 2008, 7, 988-1000. [CrossRef]

42. Pegg, G.F.; Brady, B.L. Verticillium Wilts; CABI Pub.: Wallingford, Oxon, UK, 2002; ISBN 978-0-85199-529-8.

43. Zhao, P.; Zhao, Y.-L.; Jin, Y.; Zhang, T.; Guo, H.-S. Colonization Process of Arabidopsis Thaliana Roots by a Green Fluorescent Protein-Tagged Isolate of Verticillium Dahliae. Protein Cell 2014, 5, 94-98. [CrossRef]

44. Zhao, Y.-L.; Zhou, T.-T.; Guo, H.-S. Hyphopodium-Specific VdNoxB/VdPls1-Dependent ROS-Ca ${ }^{2+}$ Signaling Is Required for Plant Infection by Verticillium Dahliae. PLoS Pathog. 2016, 12, e1005793. [CrossRef]

45. Ali, M.; Cheng, Z.; Ahmad, H.; Hayat, S. Reactive Oxygen Species (ROS) as Defenses against a Broad Range of Plant Fungal Infections and Case Study on ROS Employed by Crops against Verticillium Dahliae Wilts. J. Plant Interact. 2018, 13, 353-363. [CrossRef] 
46. Papaioannou, I.A.; Ligoxigakis, E.K.; Vakalounakis, D.J.; Markakis, E.A.; Typas, M.A. Phytopathogenic, Morphological, Genetic and Molecular Characterization of a Verticillium Dahliae Population from Crete, Greece. Eur. J. Plant Pathol. 2013, 136, 577-596. [CrossRef]

47. Vangalis, V.; Papaioannou, I.A.; Markakis, E.A.; Knop, M.; Typas, M.A. Hex1, the Major Component of Woronin Bodies, Is Required for Normal Development, Pathogenicity, and Stress Response in the Plant Pathogenic Fungus Verticillium Dahliae. J. Fungi. 2020, 6, 344. [CrossRef]

48. Nguyen, Q.B.; Kadotani, N.; Kasahara, S.; Tosa, Y.; Mayama, S.; Nakayashiki, H. Systematic Functional Analysis of CalciumSignalling Proteins in the Genome of the Rice-Blast Fungus, Magnaporthe Oryzae, Using a High-Throughput RNA-Silencing System. Mol. Microbiol. 2008, 68, 1348-1365. [CrossRef] [PubMed]

49. Paz, Z.; García-Pedrajas, M.D.; Andrews, D.L.; Klosterman, S.J.; Baeza-Montañez, L.; Gold, S.E. One Step Construction of Agrobacterium-Recombination-Ready-Plasmids (OSCAR), an Efficient and Robust Tool for ATMT Based Gene Deletion Construction in Fungi. Fungal Genet. Biol. 2011, 48, 677-684. [CrossRef]

50. Lu, S.; Lyngholm, L.; Yang, G.; Bronson, C.; Yoder, O.C.; Turgeon, B.G. Tagged Mutations at the Tox1 Locus of Cochliobolus Heterostrophus by Restriction Enzyme-Mediated Integration. Proc. Natl. Acad. Sci. USA 1994, 91, 12649-12653. [CrossRef] [PubMed]

51. Markakis, E.A.; Ligoxigakis, E.K.; Avramidou, E.V.; Tzanidakis, N. Survival, Persistence, and Infection Efficiency of Verticillium Dahliae Passed Through the Digestive System of Sheep. Plant Dis. 2014, 98, 1235-1240. [CrossRef] [PubMed]

52. Markakis, E.A.; Fountoulakis, M.S.; Daskalakis, G.C.; Kokkinis, M.; Ligoxigakis, E.K. The Suppressive Effect of Compost Amendments on Fusarium Oxysporum f.Sp. Radicis-Cucumerinum in Cucumber and Verticillium Dahliae in Eggplant. Crop. Prot. 2016, 79, 70-79. [CrossRef]

53. Livak, K.J.; Schmittgen, T.D. Analysis of Relative Gene Expression Data Using Real-Time Quantitative PCR and the 2(-Delta Delta C(T)) Method. Methods 2001, 25, 402-408. [CrossRef]

54. Vangalis, V.; Likhotkin, I.; Knop, M.; Typas, M.A.; Papaioannou, I.A. Starvation-Induced Cell Fusion and Heterokaryosis Frequently Escape Imperfect Allorecognition Systems to Enable Parasexual Interactions in an Asexual Fungal Pathogen. bioRxiv 2021. [CrossRef]

55. Rodrigues-Pousada, C.; Devaux, F.; Caetano, S.M.; Pimentel, C.; da Silva, S.; Cordeiro, A.C.; Amaral, C. Yeast AP-1 like Transcription Factors (Yap) and Stress Response: A Current Overview. Microb. Cell 2019, 6, 267-285. [CrossRef] [PubMed]

56. Huang, J.; Canadien, V.; Lam, G.Y.; Steinberg, B.E.; Dinauer, M.C.; Magalhaes, M.A.O.; Glogauer, M.; Grinstein, S.; Brumell, J.H. Activation of Antibacterial Autophagy by NADPH Oxidases. Proc. Natl. Acad. Sci. USA 2009, 106, 6226-6231. [CrossRef] [PubMed]

57. Hutchison, E.; Brown, S.; Tian, C.; Glass, N.L. Transcriptional Profiling and Functional Analysis of Heterokaryon Incompatibility in Neurospora Crassa Reveals That Reactive Oxygen Species, but Not Metacaspases, Are Associated with Programmed Cell Death. Microbiology (Reading) 2009, 155, 3957-3970. [CrossRef] [PubMed]

58. Mehta, N.; Patil, R.; Baghela, A. Differential Physiological Prerequisites and Gene Expression Profiles of Conidial Anastomosis Tube and Germ Tube Formation in Colletotrichum Gloeosporioides. J. Fungi 2021, 7, 509. [CrossRef]

59. Marschall, R.; Tudzynski, P. Reactive Oxygen Species in Development and Infection Processes. Semin. Cell Dev. Biol. 2016, 57, 138-146. [CrossRef]

60. Kayano, Y.; Tanaka, A.; Akano, F.; Scott, B.; Takemoto, D. Differential Roles of NADPH Oxidases and Associated Regulators in Polarized Growth, Conidiation and Hyphal Fusion in the Symbiotic Fungus Epichloë Festucae. Fungal Genet. Biol. 2013, 56, 87-97. [CrossRef]

61. Sun, L.; Qin, J.; Rong, W.; Ni, H.; Guo, H.-S.; Zhang, J. Cellophane Surface-Induced Gene, VdCSIN1, Regulates Hyphopodium Formation and Pathogenesis via CAMP-Mediated Signalling in Verticillium Dahliae. Mol. Plant Pathol. 2019, 20, 323-333. [CrossRef] [PubMed]

62. Yu, J.; Li, T.; Tian, L.; Tang, C.; Klosterman, S.J.; Tian, C.; Wang, Y. Two Verticillium Dahliae MAPKKKs, VdSsk2 and VdSte11, Have Distinct Roles in Pathogenicity, Microsclerotial Formation, and Stress Adaptation. Msphere 2019, 4, e00426-19. [CrossRef] [PubMed]

63. Li, X.; Kang, Y.-Q.; Luo, Y.-L.; Zhang, K.-Q.; Zou, C.-G.; Liang, L.-M. The NADPH Oxidase AoNoxA in Arthrobotrys Oligospora Functions as an Initial Factor in the Infection of Caenorhabditis Elegans. J. Microbiol. 2017, 55, 885-891. [CrossRef] [PubMed]

64. Kicka, S.; Bonnet, C.; Sobering, A.K.; Ganesan, L.P.; Silar, P. A Mitotically Inheritable Unit Containing a MAP Kinase Module. Proc. Natl. Acad. Sci. USA 2006, 103, 13445-13450. [CrossRef] [PubMed]

65. Dikalov, S. Crosstalk between Mitochondria and NADPH Oxidases. Free Radic. Biol. Med. 2011, 51, 1289-1301. [CrossRef] [PubMed]

66. Riquelme, M.; Aguirre, J.; Bartnicki-García, S.; Braus, G.H.; Feldbrügge, M.; Fleig, U.; Hansberg, W.; Herrera-Estrella, A.; Kämper, J.; Kück, U.; et al. Fungal Morphogenesis, from the Polarized Growth of Hyphae to Complex Reproduction and Infection Structures. Microbiol. Mol. Biol. Rev. 2018, 82, e00068-17. [CrossRef] [PubMed]

67. Fleissner, A.; Leeder, A.C.; Roca, M.G.; Read, N.D.; Glass, N.L. Oscillatory Recruitment of Signaling Proteins to Cell Tips Promotes Coordinated Behavior during Cell Fusion. Proc. Natl. Acad. Sci. USA 2009, 106, 19387-19392. [CrossRef] [PubMed]

68. Paul, S.; Doering, T.L.; Moye-Rowley, W.S. Cryptococcus Neoformans Yap1 Is Required for Normal Fluconazole and Oxidative Stress Resistance. Fungal Genet. Biol. 2015, 74, 1-9. [CrossRef] 
69. Peng, C.A.; Gaertner, A.A.E.; Henriquez, S.A.; Fang, D.; Colon-Reyes, R.J.; Brumaghim, J.L.; Kozubowski, L. Fluconazole Induces ROS in Cryptococcus Neoformans and Contributes to DNA Damage in Vitro. PLoS ONE 2018, 13, e0208471. [CrossRef]

70. Lev, S.; Hadar, R.; Amedeo, P.; Baker, S.E.; Yoder, O.C.; Horwitz, B.A. Activation of an AP1-Like Transcription Factor of the Maize Pathogen Cochliobolus Heterostrophus in Response to Oxidative Stress and Plant Signals. Eukaryot. Cell 2005, 4, $443-454$. [CrossRef]

71. Lin, C.-H.; Yang, S.L.; Chung, K.-R. The YAP1 Homolog-Mediated Oxidative Stress Tolerance Is Crucial for Pathogenicity of the Necrotrophic Fungus Alternaria Alternata in Citrus. Mol. Plant Microbe Interact. 2009, 22, 942-952. [CrossRef]

72. Tang, C.; Li, T.; Klosterman, S.J.; Tian, C.; Wang, Y. The BZIP Transcription Factor VdAtf1 Regulates Virulence by Mediating Nitrogen Metabolism in Verticillium Dahliae. New Phytol. 2020, 226, 1461-1479. [CrossRef]

73. Tang, C.; Xiong, D.; Fang, Y.; Tian, C.; Wang, Y. The Two-Component Response Regulator VdSkn7 Plays Key Roles in Microsclerotial Development, Stress Resistance and Virulence of Verticillium Dahliae. Fungal Genet. Biol. 2017, 108, 26-35. [CrossRef] [PubMed]

74. Wang, Y.; Tian, L.; Xiong, D.; Klosterman, S.J.; Xiao, S.; Tian, C. The Mitogen-Activated Protein Kinase Gene, VdHog1, Regulates Osmotic Stress Response, Microsclerotia Formation and Virulence in Verticillium Dahliae. Fungal Genet. Biol. 2016, 88, 13-23. [CrossRef]

75. Vivancos, A.P.; Castillo, E.A.; Biteau, B.; Nicot, C.; Ayté, J.; Toledano, M.B.; Hidalgo, E. A Cysteine-Sulfinic Acid in Peroxiredoxin Regulates H2O2-Sensing by the Antioxidant Pap1 Pathway. Proc. Natl. Acad. Sci. USA 2005, 102, 8875-8880. [CrossRef] [PubMed]

76. Vargas-Pérez, I.; Sánchez, O.; Kawasaki, L.; Georgellis, D.; Aguirre, J. Response Regulators SrrA and SskA Are Central Components of a Phosphorelay System Involved in Stress Signal Transduction and Asexual Sporulation in Aspergillus Nidulans. Eukaryot. Cell 2007, 6, 1570-1583. [CrossRef] [PubMed]

77. Jaimes-Arroyo, R.; Lara-Rojas, F.; Bayram, Ö.; Valerius, O.; Braus, G.H.; Aguirre, J. The SrkA Kinase Is Part of the SakA MitogenActivated Protein Kinase Interactome and Regulates Stress Responses and Development in Aspergillus Nidulans. Eukaryot. Cell 2015, 14, 495-510. [CrossRef] [PubMed]

78. Garrido-Bazán, V.; Jaimes-Arroyo, R.; Sánchez, O.; Lara-Rojas, F.; Aguirre, J. SakA and MpkC Stress MAPKs Show Opposite and Common Functions During Stress Responses and Development in Aspergillus Nidulans. Front. Microbiol. $2018,9,2518$. [CrossRef] 